\title{
Phylogenetic toric varieties on graphs
}

\author{
Weronika Buczyńska
}

Received: 19 November 2010 / Accepted: 31 July 2011 / Published online: 7 September 2011

(C) The Author(s) 2011. This article is published with open access at Springerlink.com

\begin{abstract}
We define phylogenetic projective toric model of a trivalent graph as a generalization of a binary symmetric model of a trivalent phylogenetic tree. Generators of the projective coordinate ring of the models of graphs with one cycle are explicitly described. The phylogenetic models of graphs with the same topological invariants are deformation-equivalent and share the same Hilbert function. We also provide an algorithm to compute the Hilbert function.
\end{abstract}

Keywords Binary symmetric model · GIT quotient · Hilbert function

\section{Introduction and background}

The inspiration for this work are toric varieties arising in computational biology, or more precisely in phylogenetic algebraic geometry. The references to the subject include [5, 16] and [18].

Markov models on phylogenetic trees are statistical models describing evolution. They are usually defined as a subset of the probability simplex, parametrized by a subset of matrices depending on the model. Among them there are group-based models on phylogenetic trees. These are special, as their projective versions, that is, the Zariski closure of the parametrization in the complex projective space, are projective toric varieties.

We are interested in the simplest group-based models-binary symmetric models, also called the Jukes-Cantor models, on trivalent trees. The object of study is the generalization of those models to trivalent graphs.

This is a shortened version of author's Ph.D. thesis.

W. Buczyńska (凶)

Department of Mathematics, Texas A\&M University, College Station, TX 77843, USA

e-mail: wkrych@mimuw.edu.pl 
The article is organized in the following way: in Sect. 1.1, we give motivation to the subject and point out references where the main object of our study appears. Then we give a brief introduction to our main tools: we recall geometric invariant theory in Sect. 1.3, next, in Sect. 1.4 we set a notation for projective toric varieties and in Sect. 1.5 provide a description of GIT quotient of a projective toric variety by subtorus of its big torus.

In Sect. 2.1 we state combinatorial relations between the topological invariants of a trivalent graph and we prove that graphs with the same invariants are mutationequivalent. In Sect. 2 we define the model of a trivalent graph as a GIT quotient of a product of $\mathbb{P}^{3}$ 's indexed by the inner vertices of the graph. This implies that the model is toric and comes with an embedding into a weighted projective space. Our first result is Theorem 2.29 that lists the set of minimal generators of the projective coordinate ring of the model, when the underlying graph has the first Betti number at most one. Section 3 contains our second result-models of mutation-equivalent graphs are deformation-equivalent.

Theorem 3.5 Geometric models of connected trivalent graphs with $n$ leaves and the first Betti number $g$ are deformation-equivalent in the projective toric variety $\mathbb{P}_{g, n}$, which is a quotient of $\mathbb{P}^{2^{n+2 g-1}-1}$ by a $g$-dimensional torus. Moreover, the deformations are invariant with respect to the action of the torus $\mathbb{T}(\mathcal{L})$ corresponding to the leaves of the graph.

In Sect. 4 we prove that the Hilbert functions of mutation-equivalent models are equal (Theorem 4.5) and finally we compute these Hilbert functions explicitly.

\subsection{Motivation-Markov models on phylogenetic trees}

A phylogenetic tree is an acyclic connected graph with additional data attached to its edges and vertices. At a vertex $v$ there is a finite ordered set $A_{v}$ called an alphabet. At an edge with ends $v$ and $w$ there is a doubly stochastic matrix (all rows and columns sums are 1) with the $(i, j)$ th entry indicating the probability of the $i$ th letter of $A_{v}$ being changed to the $j$ th letter $A_{w}$. To construct a Markov model on a phylogenetic tree we first need to indicate a set of observable vertices. Then the model is the subvariety of the probability simplex, parametrized by a set of matrices: their entries are probabilities of observing letters at the observable vertices. We consider symmetric models, which means the matrices are symmetric. Typically the observable vertices are the leaves of the tree.

Apart form this real variety, one can consider its complex algebraic relaxation. That is, the parameters are allowed to vary in a complex projective space and we take the Zariski closure of the image. Then the model becomes a complex projective variety and can be studied by means of algebraic geometry. Binary symmetric models have additional structure-they are equipped with an action of a torus of dimension equal to the dimension of the model and thus they are projective toric varieties. This is an especially nice class of varieties, which has a combinatorial description by lattice polytopes. The geometry of the simplest group-based models-binary symmetric models with the restriction that the underlying tree is trivalent was the object of study 
of [3]. In that paper we described the corresponding lattice polytope and interpreted the models as a certain quotient of a product of three-dimensional projective spaces.

\subsection{Toric algebras of our graph models in the literature}

We generalize the quotient description of the tree models introduced in [3] and we get a toric projective model, which this time is embedded in a weighted projective space. Such an embedding is always given by a graded lattice cone. We denote the cone for a graph $\mathcal{G}$ by $\tau(\mathcal{G})$.

The way we associate a lattice cone to a trivalent graph appears in the Jeffrey and Weitsmann's [10] study of moduli of flat $S U(2)$-connections on a genus $g$ Riemann surface. In this context the trivalent graph $\mathcal{G}$ describes the geometry of the compact surface $\Sigma^{g}$ of genus $g$ and thus has no leaves. A subset of $\mathbb{Z}$-labelings of the graph, which are exactly points of our cone $\tau(\mathcal{G})$, are in 1-1 correspondence with the number of Bohr-Sommerfeld fibers which is the central object of study in [10]. By the Verlinde formula, the number of those fibers equals the dimension of holomorphic sections of powers of a natural line bundle on the moduli space of flat $S U(2)$ connections on $\Sigma^{g}$. This number is a value of the Hilbert function of the toric model of a connected graph with no leaves and the first Betti number $g$. The varieties $X(\mathcal{G})$ appear naturally in the algebraic and symplectic geometry of the above mentioned moduli space of flat $S U$ (2)-connections, as discussed by Manon in [11] and in [12].

By our Theorem 4.5, we know that the Hilbert function only depends on the topological invariants of the graph.

Although the model depends on the shape of the underlying trivalent tree, once we restrict ourselves to trees with fixed number of leaves, models of all of them are in the same irreducible component of the Hilbert scheme of projective varieties with fixed Hilbert polynomial. This was proved by Sturmfels and Xu in [20]. This direction was extended and continued by Manon [11]. He constructs a sheaf of algebras over the moduli stack $\overline{\mathcal{M}}_{g, n}$ of genus $g, n$-pointed stable curves, whose fiber over a curve is the Cox ring of the moduli of quasi-parabolic principal vector bundles associated to a simple reductive group. Our semigroup algebras $\mathbb{C}[\tau(\mathcal{G})]$ are obtained by some initial term deformations from algebras above the most special points of $\mathcal{M}_{g, n}$ in Manon's construction.

Any trivalent graph is made by gluing together tripods, that is, graphs $入$ with four vertices and three edges attached to the central vertex. To construct the toric model we assign to every inner vertex a copy of a three-dimensional complex projective space and to every edge we assign an action of the one-dimensional complex torus $\mathbb{C}^{*}$ on the product of all those $\mathbb{P}^{3}$, which corresponds to gluing two tripods along that edge. The model $X(\mathcal{G})$ of the trivalent graph $\mathcal{G}$ is a geometric invariant theory (GIT) quotient of product of the $\mathbb{P}^{3}$ by the torus defined as a product of the $\mathbb{C}^{*}$ 's corresponding to the internal edges. We also translate this description into language of projective toric varieties, by writing the model $X(\mathcal{G})$ as the projective spectrum of a semigroup ring $\mathbb{C}[\tau(\mathcal{G})]$. The underlying semigroup $\tau(\mathcal{G})$ has a clear description in terms of the graph $\mathcal{G}$.

Three results of this article generalize our earlier results obtained in [3] about binary symmetric models of trivalent trees to phylogenetic graph models. First we 
describe the minimal $\mathbb{Z}$-generators of the semigroup $\tau(\mathcal{G})$ when the graph $\mathcal{G}$ has the first Betti number at most one. We also prove that models of graphs with the same discrete invariants are deformation-equivalent and lastly that they share the same Hilbert function.

\subsection{Geometric invariant theory}

We use geometric invariant theory for a normal projective variety $X$ with an action of an algebraic torus $\mathbb{T}$. Our main reference is Sects. 5 and 6 of [1], although the setup we use may seem to be slightly more general than the one found in [1]. Namely, instead of linearizing only with respect to a line bundle we allow ample Weil divisors. We will explain the necessary modifications and show how this does not affect the basic theory.

Definition 1.1 A divisor $\mathcal{L}$ is an ample Weil divisor if some positive multiple $n \mathcal{L}$ is an ample line bundle.

Given an ample Weil divisor $\mathcal{L}$ we have the ring

$$
R(X, \mathcal{L}):=\bigoplus_{p=0}^{\infty} H^{0}(X, \mathcal{O}(p \mathcal{L}))
$$

which is the projective coordinate ring of $X$ embedded into a weighted projective space by the linear system $|\mathcal{L}|$. This is completely analogous, see [17], to the standard way of describing embedding of $X$ into a projective space with a very ample line bundle $\mathcal{L}$, see [9, Sect. II.2]. We discuss these facts in Sect. 1.4.

We denote by

$$
R_{p}(X, \mathcal{L}):=R(X, p \mathcal{L})
$$

the ring given by a multiple of $\mathcal{L}$. When the divisor $\mathcal{L}$ is clear, we write $R(X)$ and $R_{p}(X)$ an instead of $R(X, \mathcal{L})$ and $R(X, p \mathcal{L})$.

Lemma 1.2 (Veronese embedding) Let $X=\operatorname{Proj} R(X, \mathcal{L})$ be a projective variety with a distinguished ample Weil divisor $\mathcal{L}$. Then for any positive integer $p$ the inclusion $R_{p}(X, \mathcal{L}) \hookrightarrow R(X, \mathcal{L})$ induces an isomorphism

$$
\operatorname{Proj} R(X, \mathcal{L}) \simeq \operatorname{Proj} R_{p}(X, \mathcal{L}) .
$$

Proof See [7, Chap. 2, Theorem 2.4.7], and also [9, Example II 5.13].

We briefly recall the definitions of quotients from [1] simplifying the statements to match our case. A map $\pi: X \rightarrow Y$ is affine if preimage of any affine subset of $Y$ is affine. Let $G$ be a reductive algebraic group acting on a variety $X$. A $G$-equivariant morphism $\pi: X \rightarrow Y$ is called a semi-geometric quotient if

- the image of every closed orbit is closed, and this property is invariant under base change 
- $\pi$ is surjective and images of disjoint, closed orbits are disjoint and this property is invariant under base change

- $\pi_{*}\left(\mathcal{O}_{X}^{G}\right)=\mathcal{O}_{Y}$.

The map $\pi: X \rightarrow Y$ is called a good quotient if it is both affine and semi-geometric.

This definition implies that the good quotient is a categorical quotient, and thus unique [1, Remark 3.1 and Theorem 3.2].

To construct the quotient, we need some more definitions.

Definition 1.3 Let $G$ be a reductive algebraic group acting on $X$. An ample Weil divisor $\mathcal{L}$ on $X$ is $G$-linearized if the action of $G$ can be lifted to the projective coordinate ring $R(X, \mathcal{L})$. More precisely, the action of $G$ on $X=\operatorname{Proj} R(X, \mathcal{L})$ induced by the lift, is the action of $G$ on $X$.

When $\mathcal{L}$ is a line bundle, a $G$-linearization is an action of $G$ on $\mathcal{L}$ which is linear on fibers and agrees with its action on $X$. We recall the definition of an $\mathcal{L}$-semistable point $[1, \S 6.1]$, which is independent of the multiple of $\mathcal{L}$.

Definition 1.4 Let $\mathcal{L}$ be an ample Weil divisor. A point $x \in X$ is $\mathcal{L}$-semistable if there exists a $G$-invariant section of a positive multiple of $\mathcal{L}$ that does not vanish at $x$. We denote by $X^{s s}$ the set of all semistable points. The GIT quotient of $X$ by $G$ with respect to the linearization $\mathcal{L}$ is

$$
X / / G=X^{s s} / / G
$$

where $X^{s s} / / G$ denotes the good quotient of the set of semistable points with respect to $\mathcal{L}$ by the action of $G$. The rational map $X \rightarrow X / / G$ is called GIT quotient map and the regular map $X^{s s} \rightarrow X / / G$ is a good quotient map.

Remark 1.5 Both [13] and [1] assume that the section in the above definition has an affine support, but as we consider only ample $\mathcal{L}$ all its sections automatically have affine support.

The following theorem says that in the above situation a GIT quotient exists and is the projective spectrum of the ring of invariants.

Theorem 1.6 Let $G$ be a reductive group acting on projective varieties $X$ and $Y$.

(i) Let $\mathcal{L}$ be a $G$-linearized ample Weil divisor on $X$. Then

$$
X / / G=\operatorname{Proj} R(X, \mathcal{L})^{G} .
$$

(ii) Let $X \hookrightarrow Y$ be a $G$-equivariant embedding, with $Y=\operatorname{Proj} R$ and $X=\operatorname{Proj} R / I$, where $I$ is the homogeneous ideal of $X$ in $Y$. Then $I$ is generated by invariants $f_{1}, \ldots, f_{j}$, the map $X / / G \hookrightarrow Y / / G$ is an embedding and the homogeneous ideal of $X / / G$ in $R^{G}$ is also generated by $f_{1}, \ldots, f_{j}$. 
Proof In [1, §5] the affine quotient is defined for an affine variety $X$ as a spectrum of invariants and [1, Theorem 5.4] says it is a good quotient. Then [1, §7] provides a characterization of a good quotient $\pi: X \rightarrow Y$ as a locally affine quotient. Equivalently, the map $\pi$ is a good quotient if and only if for any open affine subset $U \subset Y$ the restriction of the map $\pi$ to $\pi^{-1}(U)$ is an affine quotient.

We know from [1, Theorem 6.2.1] that (i) is true for projective space and $\mathcal{L}=$ $\mathcal{O}(1)$. Additionally for an arbitrary variety $X=\operatorname{Proj} R$ and a very ample line bundle $\mathcal{L}$ that defines an equivariant embedding into $\mathbb{P}^{n}$ by $[1, \S 6.3]$ we have:

- $X^{s s}=\left(\mathbb{P}^{n}\right)^{s s} \cap X$

- the restriction of the good quotient morphism $\pi:\left(\mathbb{P}^{n}\right)^{s s} \rightarrow\left(\mathbb{P}^{n}\right)^{s s} / / G$ is the good quotient morphism on $X$.

We choose an affine covering $\mathcal{U}$ of the quotient $\mathbb{P}^{n} / / G$ by sets of the form $\{f \neq 0\}$, where $f$ is $G$-invariant. Then $\left\{\pi^{-1}(U) \mid U \in \mathcal{U}\right\}$ is an affine $G$-invariant covering of $\mathbb{P}^{n}$, since $\pi$ is an affine map. By [1, Theorem 5.3] and the Hilbert-Nagata theorem [1, Theorem 5.2] for each $U \in \mathcal{U}$ we know that $\left(\pi^{-1}(U) \cap X\right) / / G$ is a spectrum of invariants

$$
\left(\pi^{-1}(U) \cap X\right) / / G=\operatorname{Spec}\left(R\left[f^{-1}\right]^{0}\right)^{G} .
$$

Also each $\pi^{-1}(U) \cap X$ has form $\left\{g=\left.\pi^{*} f\right|_{X} \neq 0\right\}$. To see that $X / / G=\operatorname{Proj} R^{G}$, we only need to see that $\left(R^{G}\left[g^{-1}\right]\right)^{0}=\left(R\left[g^{-1}\right]^{0}\right)^{G}$, which is true since $g$ is $G$-invariant. Thus (i) holds for $X$ projective and $\mathcal{L}$ very ample.

When $\mathcal{L}$ is an ample Weil divisor, we use Lemma 1.2 to replace $\mathcal{L}$ by its $k$ th power. More precisely, we choose $k$ such that $k \mathcal{L}$ is a very ample line bundle, and since the set of semistable points does not depend on the multiple of $\mathcal{L}$ we have

$$
X / / G=\operatorname{Proj} R_{p}(X, \mathcal{L})^{G}=\operatorname{Proj}\left(R(X, \mathcal{L})^{G}\right)_{p}=\operatorname{Proj} R(X, \mathcal{L})^{G}
$$

where the first equality holds, because $k \mathcal{L}$ is very ample, the second holds because the action of $G$ preserves gradations, and in the third we use Lemma 1.2.

For the proof of (ii) by Hilbert-Nagata theorem, we know that $I$ is generated by invariants. Since a good quotient is a locally affine quotient the map $X / / G \rightarrow Y / / G$ is an embedding. The last part of (ii) follows from [1, Theorem 5.2].

\subsection{Toric varieties in weighted projective spaces}

The embedding of a projective toric variety $X$ into a projective space is described by a polytope $\Delta$ with integral vertices. If we scale the polytope by an integer $k$, then we do not change the variety. The resulting embedding changes by composing it with $k$ th Veronese embedding. The sum of all positive multiples forms a semigroup (or a graded lattice cone). In this situation $X=\operatorname{Proj} \bigoplus_{k \in \mathbb{N}} \mathbb{C}[k \Delta]$. When the ambient space is a weighted projective space the embedding is given by a graded cone with a set of (minimal) $\mathbb{Z}$-generators, which are no longer in the first degree. Every section of this cone determined by the grading is a rational polytope.

Definition 1.7 A weighted projective space $\mathbb{P}\left(a_{0}, \ldots, a_{n}\right)$ with weights $\left(a_{0}, \ldots, a_{n}\right)$ where each $a_{i} \in \mathbb{N}$ is a positive integer is the GIT quotient of the affine space $\mathbb{C}^{n+1}$ 
by the action

$$
t \cdot\left(x_{0}, \ldots, x_{n}\right)=\left(t^{a_{0}} \cdot x_{0}, \ldots, t^{a_{n}} \cdot x_{n}\right) .
$$

Again, the non-stable locus is the point $0 \in \mathbb{C}^{n+1}$.

We can assume that the greatest common divisor of the weights is one-this is by substitution $t \mapsto t^{\operatorname{gcd}\left(a_{0}, \ldots, a_{n}\right)}$. Let us pick integers $b_{0}, \ldots, b_{n}$ such that $\sum_{i=0}^{n} b_{i}$. $a_{i}=1$. The weighted projective space has the sheaf $\mathcal{O}_{\mathbb{P}}(1)$, which corresponds to the ample Weil divisor $\sum_{i=0}^{n} b_{i} \cdot\left(x_{i}\right)$, where the $\left(x_{i}\right)$ 's are the divisors corresponding to the coordinates.

Weighted projective spaces are often singular. They have quotient singularities coming from finite abelian group actions.

Definition 1.8 To a lattice $M$, we associate in a non-unique way a graded lattice $M^{\mathrm{gr}}$, equipped with degree map deg: $M^{\text {gr }} \rightarrow \mathbb{Z}$-the projection to the first coordinate. It fits into the exact sequence

$$
0 \longrightarrow M \longrightarrow M^{\text {gr }} \underset{s}{\stackrel{\text { deg }}{\rightleftarrows}} \mathbb{Z} \longrightarrow 0 .
$$

We fix a splitting $s: \mathbb{Z} \rightarrow M^{\text {gr }}$ of the exact sequence. Equivalently, we pick a 0 element in $M \simeq(1, M) \subset M^{\text {gr }}$. The choice of the splitting $s$ corresponds to a choice of linearization of the action of the torus $\operatorname{Spec}\left(\mathbb{C}\left[M^{\vee}\right]\right)$ on itself.

Definition 1.9 A graded lattice cone $\tau$ is a rational, convex, polyhedral cone in a graded lattice $M^{\mathrm{gr}}$, with all elements having non-negative degree: $\operatorname{deg}(\tau) \subset \mathbb{N}$, and the zero gradation consists of one element: $\operatorname{deg}^{-1}(0) \cap \tau=0$. Convex here means that $\tau \otimes_{\mathbb{Z}} \mathbb{R}_{+} \subset M^{\mathrm{gr}} \otimes_{\mathbb{Z}} \mathbb{R}$ is convex. Equivalently, $\tau$ is a (saturated) sub-semigroup of the free abelian group $M^{\text {gr }}$ with finite set of $\mathbb{Z}$-generators all having positive degrees. The only element of degree zero is the neutral element.

Proposition 1.10 A toric variety $X \subset \mathbb{P}\left(a_{0}, \ldots, a_{n}\right)$ is described by its fan and an ample Weil divisor $\mathcal{O}_{X}(1)$ or equivalently by an isomorphism $X \simeq \operatorname{Proj} \mathbb{C}[\tau]$ where $\tau$ is a graded, rational, convex, polyhedral cone in a graded lattice $M^{\mathrm{gr}}$. Then $\mathbb{C}[\tau]=$ $R / I$, where $R$ is the homogeneous coordinate ring of $\mathbb{P}\left(a_{0}, \ldots, a_{n}\right)$ and $I$ is the homogeneous ideal of $X$.

Idea of the proof The correspondence between ample Weil divisors and the graded lattice cones is the following. The degree $k$ sections of the sheaf associated to the Weil divisor form the $k$ th section of the cone $\tau$. To go the other way, we have an isomorphism $X \simeq \operatorname{Proj} \mathbb{C}[\tau]$ and then the ample Weil divisor is $\mathcal{O}_{X}(1)$ - the pullback of $\mathcal{O}_{\mathbb{P}\left(a_{0}, \ldots, a_{n}\right)}(1)$ from the ambient weighted projective space $\mathbb{P}\left(a_{0}, \ldots, a_{n}\right)$.

The choice of the 0 element of $M \simeq(1, M) \subset M^{\text {gr }}$ in Definition 1.8 is a choice of linearization of the action of the torus of $X$, which extends the action of the torus on itself. 
Definition 1.11 A graded product $M_{1}^{\mathrm{gr}} \times{ }_{g} M_{2}^{\mathrm{gr}}$ of the graded lattices $M_{1}^{\mathrm{gr}}$ and $M_{2}^{\mathrm{gr}}$ is the fiber product over their degree maps, or equivalently the hyperplane $\operatorname{deg}_{1}=\operatorname{deg}_{2}$ in the product $M_{1}^{\mathrm{gr}} \times M_{2}^{\mathrm{gr}}$ :

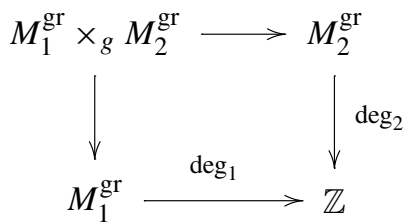

Definition 1.12 A graded product $\tau_{1} \times_{g} \tau_{2}$ of graded cones $\tau_{1}$ and $\tau_{2}$ is the fiber product over their degree map, or equivalently intersection of the hyperplane $\mathrm{deg}_{1}=$ $\operatorname{deg}_{2}$ with the product cone $\tau_{1} \times \tau_{2}$ :

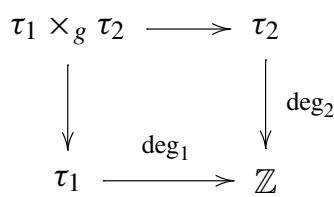

Lemma 1.13 If $\tau_{1} \subset M_{1}^{\mathrm{gr}}$ and $\tau_{2} \subset M_{2}^{\mathrm{gr}}$ are graded rational convex polyhedral cones, and

$$
X_{1}=\operatorname{Proj} \mathbb{C}\left[\tau_{1}\right], \quad X_{2}=\operatorname{Proj} \mathbb{C}\left[\tau_{2}\right]
$$

are corresponding projective toric varieties, then the product of these varieties under the Segre embedding

$$
X_{1} \times X_{2}=\operatorname{Proj} \mathbb{C}\left[\tau_{1} \times g \tau_{2}\right]
$$

corresponds to the graded product of the cones $\tau_{1}$ and $\tau_{2}$.

Proof By definition $X_{1} \times X_{2}$ under Segre embedding is equal to

$$
\operatorname{Proj} \bigoplus_{i=0}^{\infty} H^{0}\left(X_{1} \times X_{2}, \mathcal{O}_{X_{1}}(i) \otimes \mathcal{O}_{X_{2}}(i)\right)
$$

For each $i$ we know that the sections of this exterior tensor product are spanned by the product of the $i$ th graded pieces of the cones

$$
H^{0}\left(X_{1} \times X_{2}, \mathcal{O}_{X_{1}}(i) \otimes \mathcal{O}_{X_{2}}(i)\right)=\mathbb{C} \cdot\left(\left(\tau_{1} \cap \operatorname{deg}^{-1}(i)\right) \times\left(\tau_{2} \cap \operatorname{deg}^{-1}(i)\right)\right) .
$$

We conclude the lemma by summing the above equality over all $i$ 's to get

$$
\bigoplus_{i=0}^{\infty} H^{0}\left(X_{1} \times X_{2}, \mathcal{O}_{X_{1}}(i) \otimes \mathcal{O}_{X_{2}}(i)\right)=\mathbb{C}\left[\tau_{1} \times_{g} \tau_{2}\right]
$$

as required. 


\subsection{Quotient of a projective toric variety by subtorus}

We show that the quotient of a projective toric variety $X=\operatorname{Proj} \mathbb{C}[\tau]$ by a subtorus is described by an appropriate linear section of $\tau$.

We first recall some facts about toric varieties from [6]. If $X=\operatorname{Proj} \mathbb{C}[\tau]$, where $\tau \subset M^{\text {gr }}$ is a graded cone, then the torus of $X$ is given by an isomorphism $\mathbb{T}=$ $\operatorname{Hom}(M, \mathbb{Z}) \otimes_{\mathbb{Z}} \mathbb{C}^{*}$. The lattice $N=\operatorname{Hom}(M, \mathbb{Z})$ is the lattice of one-parameter subgroups of $\mathbb{T}$. For any subtorus $\mathbb{T}^{\prime}$ of the torus $\mathbb{T}$ there are corresponding maps of lattices: the projection $M \rightarrow M^{\prime}$ of the monomial lattices and the embedding $N^{\prime} \hookrightarrow N$ of the lattice of one-parameter subgroups.

A linearization of the action of the torus $\mathbb{T}$ on $X$, induces a linearization of the action of any subtorus $\mathbb{T}^{\prime} \subset \mathbb{T}$, by restricting the action. In this setting Theorem 1.6 implies the following result.

Theorem 1.14 Let $\tau$ be a graded lattice cone in a lattice $M^{\mathrm{gr}}$ and $X=\operatorname{Proj} \mathbb{C}[\tau]$ the corresponding toric variety. Let $j: \mathbb{T}^{\prime} \hookrightarrow \mathbb{T}$ be a subtorus of the torus $\mathbb{T}$, with $j_{*}: N^{\prime} \hookrightarrow N, j^{*}: M \rightarrow M^{\prime}$ and $\mathrm{id} \oplus j^{*}: M^{\mathrm{gr}} \rightarrow\left(M^{\prime}\right)^{\mathrm{gr}}$ the corresponding lattice maps. Then there exists a good quotient and it is equal to

$$
X / / \mathbb{T}^{\prime}=\operatorname{Proj} \mathbb{C}[\tau]^{\mathbb{T}^{\prime}}
$$

where

$$
\mathbb{C}[\tau]^{T^{\prime}}=\mathbb{C}\left[\tau \cap\left(\mathbb{Z} \oplus \operatorname{ker}\left(j^{*}: M \rightarrow M^{\prime}\right)\right)\right]=\mathbb{C}\left[\tau \cap\left(\mathbb{Z} \oplus N^{\prime \perp}\right)\right] .
$$

Moreover the quotient $X / / \mathbb{T}^{\prime}$ is polarized by $\mathcal{O}_{X / / \mathbb{T}^{\prime}}(1)$ in a natural way.

The following example shows that we do need ample Weil divisors, not only ample line bundles. This way the products and the quotients in the construction of the phylogenetic model commute. Without allowing ample Weil divisors, one would obtain different multiples of the initial bundle depending on the order of quotients by the $\mathbb{C}^{*}$-actions. We use this property in the course of our main proofs: while using mutations to pass to a simpler graph, in Theorem 3.5 about the deformation-equivalence to have a small ambient space, also to study the minimal generators of the associated semigroup.

Example 1.15 Let $X$ be the good GIT-quotient

$$
\pi: \mathbb{P}^{3} \times \mathbb{P}^{3} \rightarrow X=\left(\mathbb{P}^{3} \times \mathbb{P}^{3}\right) / /\left(\mathbb{C}^{*}\right)^{3}
$$

of the product of two projective three-spaces by an action of three-dimensional torus acting with weights

$$
\left[\begin{array}{ccccccccc}
(0 & 1 & 1 & 0) & \times & (0 & -1 & -1 & 0) \\
(0 & 1 & -1 & 0) & \times & (0 & 0 & 0 & 0) \\
(0 & 0 & 0 & 0) & \times & (0 & 1 & -1 & 0)
\end{array}\right]
$$

linearized with respect to the line bundle $\mathcal{O}_{\mathbb{P}^{3}}(1) \otimes \mathcal{O}_{\mathbb{P}^{3}}(1)$. We will see later that $X=X(\delta)$ is the model of the trivalent graph $\&$ with the first Betti number two with 
three edges and is a projective toric variety by Theorem 1.14 . The sheaf $\mathcal{O}_{X}(1)=$ $\pi_{*}\left(\mathcal{O}_{\mathbb{P}^{3}}(1) \otimes \mathcal{O}_{\mathbb{P}^{3}}(1)\right)$ is not a locally free $\mathcal{O}_{X}$-module because the associated divisor is not Cartier. To verify it we can use a computer algebra system, for example magma [2] as follows. Since any divisor on a toric variety is linearly equivalent to a $\mathbb{T}$-invariant divisor, we identify a divisor with a corresponding $\mathbb{Z}$-combination of primitive elements of the rays of the fan. Thus we only need to check if the $\mathbb{Z}$ combination corresponding to $\mathcal{O}_{X}(1)$ yields a piecewise linear function on the fan, which by [15] is equivalent to our $\mathbb{T}$-invariant Weil divisor being Cartier.

From the discussion in the latter part on this section, in particular Lemmas 2.21 and 2.22 , we know that

$$
X(\wp)=(X(\rho) \times X(\rho)) / / \mathbb{C}^{*} .
$$

Here $X(\rho)$ is the phylogenetic model. The quotient is equal to $\mathbb{P}(1,1,2)$ is polarized with $\mathcal{O}_{X(9)}(1)$, which only is a $\mathbb{Q}$-line bundle.

\section{Phylogenetic models on trivalent graphs}

\subsection{Trivalent graphs}

We define topological invariants of trivalent graphs. Any two graphs with the same invariants are equivalent by applying appropriate mutations, which we introduced in [3]. We do not assume that our graphs are connected.

Definition 2.1 A graph $\mathcal{G}$ is set $\mathcal{V}$ of vertices and set $\mathcal{E}$ of edges together with the unordered boundary map $\partial: \mathcal{E} \rightarrow \mathcal{V}^{\otimes 2}$, where $\mathcal{V}^{\otimes 2}$ is the set of unordered pairs of vertices. We write $\partial(e)=\left\{\partial_{1}(e), \partial_{2}(e)\right\}$ and say that $v$ is an end of the edge $e$ if $v \in \partial(e)$. A vertex incident to exactly one edge is a leaf. The set of leaves is denoted by $\mathcal{L}$ and the number of leaves by $n$. If a vertex is not a leaf, it is called an inner vertex. An edge incident to a leaf is a petiole and $\mathcal{P}$ is the set of petioles. We write comp $\mathcal{G}$ for the set of connected components of the graph and $\mid$ comp $\mathcal{G} \mid$ for the number of components. We denote by $g$ for the first Betti numbers of graph, which is the rank of the first homology group of the graph viewed as a CW-complex. A graph is trivalent if every inner vertex has valency three. Valency of a vertex $v$ is the number of connected components of a sufficiently small neighborhood of $v$ with $v$ removed. A trivalent graph with no cycles is a trivalent tree.

When discussing more than one graph we will write $\mathcal{V}(\mathcal{G}), \mathcal{E}(\mathcal{G}), n(\mathcal{G})$, etc. instead of $\mathcal{V}, \mathcal{E}, n$, etc.

We give names to the three small graphs: Dumbbell is the graph $\$$ with three edges, two loops and no leaves; LittleMan is the graph 9 with four edges, one loop and two leaves; Hammock is the graph $\_$with four edges, one 2-cycle and two leaves.

Remark 2.2 Our graphs are not oriented, nevertheless we write $\partial_{1}(e)$ and $\partial_{2}(e)$ for the vertices adjacent to the edge $e$. This makes it easier to talk about "the other end of $e^{\prime}$. 
Fig. 1 Building a trivalent graph from tripods

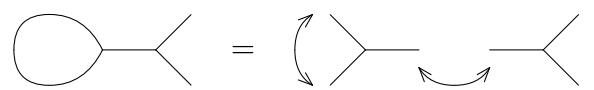

We call the unique trivalent tree $\lambda$ with a single inner vertex and three leaves the elementary tripod. It has three edges $e_{1}, e_{2}, e_{3}$. Any trivalent graph is built of elementary tripods in the following way: given a trivalent graph $\mathcal{G}$ and any inner vertex $v \in \mathcal{V}(\mathcal{G})$ we pick a copy of elementary tripod $\lambda_{v} \simeq \lambda_{\text {and a map }} i_{v}: \lambda_{v} \rightarrow \mathcal{G}$ which sends the central vertex of $\lambda_{v}$ to $v$ and locally near $i^{-1}(v)$ is an embedding. We present the graph $\mathcal{G}$ as a disjoint union of the graphs $\lambda_{v}$ with appropriate identification of edges

$$
\mathcal{G}=\bigsqcup_{v \in \mathcal{V} \backslash \mathcal{L}} \lambda_{v} /\left\{i_{\partial_{1}(e)}^{-1}(e) \sim i_{\partial_{2}(e)}^{-1}(e)\right\}_{e \in \mathcal{E} \backslash \mathcal{P}} .
$$

This mirrors the construction of the phylogenetic model $X(\mathcal{G})$ as we will see in Definition 2.11 .

Example 2.3 In Fig. 1 we give an example of the above presentation of a trivalent graph LittleMan.

Lemma 2.4 In any trivalent graph with $n$ leaves and first Betti number $g$ the following holds:

(i) $|\mathcal{V}|,|\mathcal{E}| \geq n$,

(ii) $2|\mathcal{E}|=3|\mathcal{V}|-2 n$,

(iii) $|\mathcal{V}|-|\mathcal{E}|=|\operatorname{comp} \mathcal{G}|-g$.

Thus, any three of the numbers $|\mathcal{V}|,|\mathcal{E}|, n, g,|\operatorname{comp} \mathcal{G}|$ determine the other two.

Proof To prove (ii) let us count pairs consisting of a vertex and an adjacent edge. On one hand we will count every edge twice. On the other hand every inner vertex has three incident edges so we have $3(|\mathcal{V}|-n)$ pairs and another $n$ pairs come from leaves which totals to $3|\mathcal{V}|-2 n$. Equation (iii) counts the Euler characteristic $|\operatorname{comp} \mathcal{G}|-g$ of the graph.

Here we introduce operations of gluing two leaves of a graph, cutting an edge into two new edges and taking a disjoint sum of two graphs.

Definition 2.5 We will use the following three constructions of trivalent graphs.

- $\mathcal{G} \sqcup \mathcal{G}^{\prime}$ is the disjoint sum of the given graphs $\mathcal{G}$ and $\mathcal{G}^{\prime}$.

- $\mathcal{G}_{l_{2}}^{l_{1}} \supset$ is the graph obtained from a given graph $\mathcal{G}$ with two distinguished leaves $l_{1}, l_{2} \in \mathcal{L}(\mathcal{G})$ by gluing the two leaves $l_{1}$ and $l_{2}$, or more precisely by removing $l_{1}$ and $l_{2}$ and identifying the edge incident to $l_{1}$ with the edge incident to $l_{2}$.

- $\mathcal{G}_{l} \star \mathcal{G}_{l^{\prime}}^{\prime}$ a graft of given graphs $\mathcal{G}$ and $\mathcal{G}^{\prime}$ each with a distinguished leaf. Figure 2 is a schematic picture of this construction. 
Fig. 2 Graft of two graphs

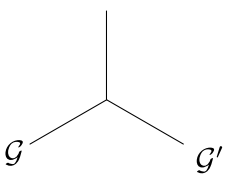

Fig. 3 Cycle edge, cycle leg and cycle vertex of a graph

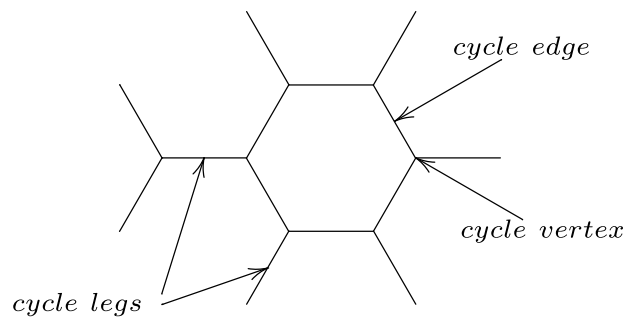

Fig. 4 A path, a cycle and a network containing a loop
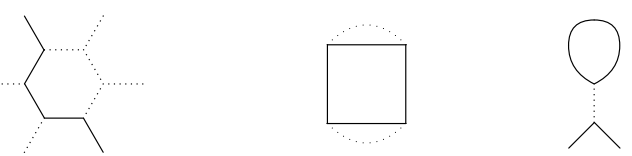

The new graph can be written as

$$
\mathcal{G}_{l} \star \mathcal{G}_{l^{\prime}}^{\prime}=\left(\left(\left(\mathcal{G} \sqcup \mathcal{G}^{\prime}\right)_{e_{1}}^{l} \supset\right) \sqcup \mathcal{G}^{\prime}\right)_{e_{2}}^{l^{\prime}} \supset .
$$

- $\mathcal{G}^{e}$ is the graph obtained from the given graph $\mathcal{G}$ by cutting an internal edge $e \in$ $\mathcal{E}(\mathcal{G}) \backslash \mathcal{L}(\mathcal{G})$. More precisely we replace $e$ with two new edges $e_{1}$ and $e_{2}$ so that $\partial_{1}\left(e_{1}\right):=\partial_{1}(e)$ and $\partial_{1}\left(e_{2}\right):=\partial_{2}(e)$. There are two new leaves in $\mathcal{G}^{e}$, which are the free ends $\partial_{2}\left(e_{1}\right)$ and $\partial_{2}\left(e_{2}\right)$ of $e_{1}$ and $e_{2}$, respectively.

Definition 2.6 An edge $e \in \mathcal{E}$ is called a cycle edge if it is not a petiole and removing it does not disconnect the connected component of the graph that contains $e$. An edge $e \in \mathcal{E}$ is called cycle leg if it is incident to a cycle edge but is not a cycle edge itself. A vertex $v \in \mathcal{V}$ is called cycle vertex if it is an end of a cycle edge. We draw an example of those in Fig. 3.

A path is a sequence of distinct edges $e_{0}, \ldots, e_{m}$ with $\partial_{2}\left(e_{i}\right)=\partial_{1}\left(e_{i+1}\right)$ for all $i \in\{0, \ldots, m-1\}$, moreover $\partial_{1}\left(e_{0}\right)$ and $\partial_{2}\left(e_{m}\right)$ are either both leaves or they are equal. In the latter case, the path is called a cycle. Paths are disjoint if they have no common vertices. A network is a union of disjoint paths. For consistency we say that the empty set is also a network. A cycle is a minimal sequence of cycle edges. A cycle of length one is a loop. In Fig. 4 we draw examples for each of those sequences.

A graph $\mathcal{G}$ is called a polygon graph if it has $2 k$ edges of which $k$ form the only cycle of $\mathcal{G}$ and the remaining $k$ edges are cycle legs. If $\mathcal{G}$ is any trivalent graph, $e \in$ $\mathcal{V}(\mathcal{G})$ its non-cycle edge, then after cutting $e$ we get a decomposition $\mathcal{G}^{e}=\mathcal{G}_{0} \sqcup \mathcal{G}_{1}$. If $\mathcal{G}_{1}$ is a tree then we call it a pendant tree. 
Fig. 5 The three trees with four labeled leaves

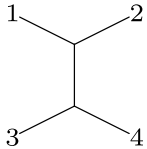

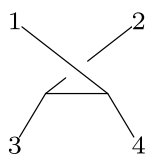

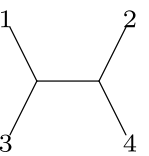

Fig. 6 Mutation along cycle edge shortens a cycle
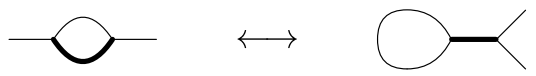

Fig. 7 Caterpillar tree

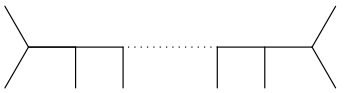

Figure 5 shows the three trivalent trees with one internal edge $e$ and four labeled leaves. Let $\mathcal{G}$ be a trivalent graph and let $e$ be an internal edge which is not a loop (the ends of $e$ are not identified). Then a neighborhood of $e$ in $\mathcal{G}$ is a trivalent tree with four leaves.

Definition 2.7 A mutation of a trivalent graph $\mathcal{G}$ along an edge $e$ is a graph $\mathcal{G}^{\prime}$ which is obtained from $\mathcal{G}$ by removing a neighborhood of $e$ (which is a trivalent tree with four leaves) and replacing it by one of the other trivalent trees from Fig. 5. Two graphs are mutation-equivalent if they may be transformed by sequence of mutations into each other.

Remark 2.8 By definition it is not possible to mutate along an edge that forms a cycle of length one. However, for longer cycles, mutations are possible, and one shortens the length of the cycle. Figure 6 shows an example of this phenomenon: Hammock graph is mutation-equivalent to LittleMan.

Lemma 2.9 Suppose edges $\left\{e_{1}, \ldots, e_{k}\right\}$ form a cycle in the graph $\mathcal{G}$ and assume $k>1$. Then, for any $i \in\{1, \ldots, k\}$ one of the two mutations along $e_{i}$ shortens the cycle $\left\{e_{1}, \ldots, e_{k}\right\}$ by one in the resulting graph, i.e. $\left\{e_{1}, \ldots, e_{i-1}, e_{i+1}, \ldots, e_{k}\right\}$ is a cycle in the new graph.

A caterpillar is a trivalent tree, which after removing all leaves and petioles becomes a string of edges as shown in Fig. 7.

Lemma 2.10 Let $\mathcal{G}_{1}$ and $\mathcal{G}_{2}$ be connected, trivalent graphs both with $n$ leaves and first Betti numbers $g$. Then they are mutation-equivalent. Moreover, for any ordered subsets $S_{1}$ of cycle edges of $\mathcal{G}_{1}$ and $S_{2}$ of cycle edges of $\mathcal{G}_{2}$, of the same size, both with the property that removing $S_{i}$ from $\mathcal{G}_{i}$ does not disconnect the graph, we can find a sequence of mutations that avoids the edges from $S_{1}\left(S_{2}\right)$ and sends ith edge of $S_{1}$ to the ith edge of $S_{2}$. Also, any mutation sends a leaf of $\mathcal{G}_{1}$ to a leaf of $\mathcal{G}_{2}$.

Proof Let $\mathcal{G}$ be connected, trivalent graph with $n$ leaves and the first Betti number $g$ and $S$ a subset of cycle edges as above. We will prove that $\mathcal{G}$ is mutation-equivalent to a trivalent graph obtained by attaching $g$ cycles of length one to a caterpillar tree 


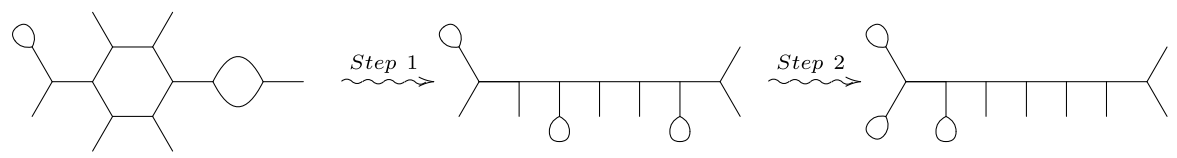

Fig. 8 Every graph is mutation-equivalent to a caterpillar graph

with $n+g$ leaves. We will choose mutations so that they will satisfy the required property.

Step 1. We proceed by induction on the set $S$ and the first Betti number of $\mathcal{G}$. For an edge $e \in S$ we can find a cycle that contains no other elements of $S$. This is because after removing all edges from $S$ the graph $\mathcal{G}$ is connected, so there is a path $\gamma$ from $\partial_{1}(e)$ to $\partial_{2}(e)$, which together with $e$ forms the required cycle. By repeatedly using Lemma 2.9, we reduce the length of this cycle to one, by performing mutations along edges from $\gamma$. In the new graph the edge $e$ forms a loop. We can consider this graph with $e$ removed, reducing both the size of $S$ and $g$. If $|S|<g$, then we repeat the above $g-|S|$ times starting from any cycle edge, which is not a loop.

After repeating this procedure $g$ times, we get a tree with $g$ loops (all edges from $S$ are among them) attached to some leaves. We can assume that this tree is a caterpillar, as we know from [3, Lemma 2.18] that any trivalent tree is mutation-equivalent to a caterpillar with the same number of leaves.

Step 2. We observe that it does not matter to which leaves the cycles are attached, we can move a cycle from a leaf to any another leaf.

In Fig. 8 we illustrate those two steps.

The last claim follows simply form the definition: mutation maps an inner edge to an inner edge, and a leaf to a leaf.

\subsection{Definition as quotient}

Given a (not necessarily connected) trivalent graph $\mathcal{G}$, we construct a toric variety $X(\mathcal{G})$, generalizing the binary symmetric model of trivalent tree from [3].

As we have already explained, see (2.1), any trivalent graph is the union of $|\mathcal{V}|-n$ elementary tripods with some edges identified. To define the variety $X(\mathcal{G})$ we replace each elementary tripod $\lambda_{v}$ with $\mathbb{P}_{v}^{3}$, union with product, and the edge identification with a quotient by an action of a one-parameter torus.

Definition 2.11 Let $\mathcal{G}$ be a trivalent graph. To an inner vertex $v \in \mathcal{V} \backslash \mathcal{L}$ we associate projective space $\mathbb{P}_{v}^{3}$ with coordinates $x_{\emptyset}^{v}, x_{12}^{v}, x_{13}^{v}, x_{23}^{v}$. To any edge $e \in \mathcal{E}$ we associate an action $\lambda_{v}^{e}$ of $\mathbb{C}^{*}$ on $\mathbb{P}_{v}^{3}$ with weights 0 and 1 as follows:

$$
\lambda_{v}^{e}(t)\left(x_{S}\right)= \begin{cases}t \cdot x_{S}^{v}, & \text { if the index of } i_{v}^{-1}(e) \in\left\{e_{1}, e_{2}, e_{3}\right\} \text { belongs to the set } S, \\ x_{S}^{v}, & \text { otherwise. }\end{cases}
$$


So we have an action of a three-dimensional torus on $\mathbb{P}^{3}=\operatorname{Proj} \mathbb{C}\left[x_{\emptyset}^{v}, x_{12}^{v}, x_{13}^{v}, x_{23}^{v}\right]$ with weights:

$$
\left[\begin{array}{llll}
0 & 1 & 1 & 0 \\
0 & 1 & 0 & 1 \\
0 & 0 & 1 & 1
\end{array}\right]
$$

For example if $i_{v}\left(e_{2}\right)=e$ then $\lambda_{v}^{e}$ acts with weight 1 on $x_{12}^{v}$ and $x_{23}^{v}$ and with weight 0 on $x_{\emptyset}^{v}$ and $x_{13}^{v}$. This action extends to an action $\lambda_{v}^{e}$ on $\prod_{v \in \mathcal{V} \backslash \mathcal{L}} \mathbb{P}_{v}^{3}$ which is non-trivial only if $v$ is an end of the edge $e$. Thus, for any internal edge $e \in \mathcal{E} \backslash \mathcal{P}$, we can define a $\mathbb{C}^{*}$-action $\lambda_{\partial_{1}(e)}^{e} \times-\lambda_{\partial_{2}(e)}^{e}$ on

$$
\prod_{v \in \mathcal{V} \backslash \mathcal{L}} \mathbb{P}_{v}^{3}
$$

to be the product action of the action $\lambda_{\partial_{1}(e)}^{e}$ on $\mathbb{P}_{\partial_{1}(e)}^{3}$ and the action $\lambda_{\partial_{2}(e)}^{e}$ with opposite weights on $\mathbb{P}_{\partial_{2}(e)}^{3}$. We define the phylogenetic model of a trivalent graph $\mathcal{G}$ to be the good quotient:

$$
X(\mathcal{G}):=\left(\prod_{v \in \mathcal{V} \backslash \mathcal{L}} \mathbb{P}_{v}^{3}\right) \quad / / \prod_{e \in \mathcal{E} \backslash \mathcal{P}}\left(\lambda_{\partial_{1}(e)}^{e} \times-\lambda_{\partial_{2}(e)}^{e}\right)
$$

of the toric variety $\prod_{v \in \mathcal{V} \backslash \mathcal{L}} \mathbb{P}_{v}^{3}$ by a subtorus of dimension $|\mathcal{E}|-|\mathcal{L}|$ of the torus. The subtorus by which we are dividing is a product of all the $\mathbb{C}^{*}$ 's over all internal edges of the graph $\mathcal{G}$ and the linearized line bundle is $\bigotimes_{v \in \mathcal{V}(\mathcal{G})} \mathcal{O}_{\mathbb{P}_{v}^{3}}(1)$. By Theorem 1.14. $X(\mathcal{G})$ is toric as it is the quotient of a toric variety by a subtorus.

Remark 2.12 In (2.3) the choice that we made defining the action of the torus $\left(\mathbb{C}^{*}\right) \backslash \mathcal{L}$ only depends on the choice of coordinates of the torus. If we choose different orientation of the edge $e$, then the two $\mathbb{C}^{*}$-actions $\lambda_{\partial_{1}(e)}^{e} \times-\lambda_{\partial_{2}(e)}^{e}$ and $-\lambda_{\partial_{1}(e)}^{e} \times \lambda_{\partial_{2}(e)}^{e}$ differ by composing with $t \mapsto \frac{1}{t}$.

Remark 2.13 Let $l$ be a leaf of a graph $\mathcal{G}$ and $e$ the adjacent petiole. The action $\lambda_{l}^{e}$ descends to a non-trivial action on the quotient variety $X(\mathcal{G})$ and is denoted by $\lambda^{l}$. For a subset $\mathcal{S} \subset \mathcal{L}$ of the leaves of $\mathcal{G}$ of cardinality $k$ we have an action of a $k$-dimensional torus $\mathbb{T}(\mathcal{S})$ - a product of the corresponding $\lambda^{l}$ 's.

If we set $k=|\mathcal{V}|-n$ the number of inner vertices, we can rewrite $|\mathcal{V}|-|\mathcal{E}|=$ $|\operatorname{comp} \mathcal{G}|-g$ using Lemma 2.4(ii) to get $k=(2 g-2|\operatorname{comp} \mathcal{G}|)+n$. Observe that

- $k$ is the number of the $\mathbb{P}^{3}$ 's in (2.2),

- $g$ is the first Betti number.

On the other hand, $|\mathcal{E}|-n=k+g-|\operatorname{comp} \mathcal{G}|$ is the number of inner edges which is the dimension of the torus that we divide by in (2.3). We get a variety of dimension

$$
\operatorname{dim} X(\mathcal{G})=3 k-(|\mathcal{E}|-n)=3 g-3|\operatorname{comp} \mathcal{G}|+2 n=|\mathcal{E}| .
$$




\subsection{Lattice and cone}

Given a trivalent graph $\mathcal{G}$ we construct the toric data that allow one to recover its toric model. The graded lattice, denoted by $M^{\text {gr }}$, as well as the graded cone $\tau(\mathcal{G})$ in it both have rank bigger by one than the dimension of the model $X(\mathcal{G})$. The latter is equal to the number of edges $\mathcal{E}(\mathcal{G})$.

Definition 2.14 Given a graph $\mathcal{G}$ let $\mathbb{Z} \mathcal{E}=\bigoplus \mathbb{Z} \cdot e$ be the lattice spanned by $\mathcal{E}$, and $\mathbb{Z} \mathcal{E}^{\vee}=\operatorname{Hom}(\mathbb{Z} \mathcal{E}, \mathbb{Z})$ be its dual. Elements of the lattice $\mathbb{Z} \mathcal{E}$ are formal linear combinations of the edges, forming the standard basis of $\mathbb{Z} \mathcal{E}$. The dual lattice $\mathbb{Z} \mathcal{E}^{\vee}$ comes with the dual basis $\left\{e^{*}\right\}_{e \in \mathcal{E}}$. We identify vertices of the graph $\mathcal{G}$ with certain elements of $\mathbb{Z} \mathcal{E}^{\vee}$ :

$$
v=\sum_{e \ni v} e^{*} .
$$

We also define $M=\{u \in \mathbb{Z} \mathcal{E}: \forall v \in \mathcal{V} v(u) \in 2 \mathbb{Z}\}$ and its dual $N=\operatorname{Hom}(M, \mathbb{Z})$. Then the graded lattice of the graph is

$$
M^{\mathrm{gr}}=\mathbb{Z} \oplus M,
$$

with the degree map

$$
\operatorname{deg}: M^{\mathrm{gr}}=\mathbb{Z} \oplus M \rightarrow \mathbb{Z},
$$

which is the projection to the first summand. The degree of $\omega \in M^{\mathrm{gr}}$ is $\operatorname{deg}(w)$.

If there is more than one graph in question we will write $M(\mathcal{G})$ for $M^{\mathrm{gr}}(\mathcal{G})$ etc.

Let us use the following notation for the elements of the lattice $\mathbb{Z} \mathcal{E}^{\vee}$ dual to the edges meeting at the vertex $v$

$$
a_{v}:=\left(i_{v}\left(e_{1}\right)\right)^{*}, \quad b_{v}:=\left(i_{v}\left(e_{2}\right)\right)^{*}, \quad c_{v}:=\left(i_{v}\left(e_{3}\right)\right)^{*},
$$

where $\left\{e_{1}, e_{2}, e_{3}\right\}$ are the edges of $入$ and $i_{v}: 入 \hookrightarrow \mathcal{G}$ is, as before, a map which is locally an embedding and sends the central vertex of the $\lambda$ to $v$-an inner vertex of $\mathcal{G}$. Whenever we use this notation we have a fixed presentation as in (2.1).

Given an element $\omega$ in either $\mathbb{Z} \mathcal{E}, M$ or $M^{\text {gr }}$, each of $a_{v}, b_{v}, c_{v} \in \mathbb{Z} \mathcal{E}^{\vee}$ measures the coefficient of $\omega$ at an edge incident to $v$. Then (2.4) becomes

$$
v=a_{v}+b_{v}+c_{v} .
$$

Definition 2.15 The degree of $\omega \in M^{\mathrm{gr}}$ at a vertex $v \in \mathcal{V}(\mathcal{G})$ is

$$
\operatorname{deg}_{v}(\omega):=\frac{1}{2} \cdot\left(a_{v}(\omega)+b_{v}(\omega)+c_{v}(\omega)\right) .
$$

The minimal degree of $\omega$ is

$$
\operatorname{deg}_{\min }(\omega):=\max _{v \in \mathcal{V}}\left\{\operatorname{deg}_{v}(\omega)\right\}
$$

where $\pi_{M}: M^{\mathrm{gr}} \rightarrow M$ is the projection to the second summand. 
The name minimal degree will become clear after we define the cone $\tau(\mathcal{G})$.

We identify paths and networks in $\mathcal{G}$ with elements of the lattices $M$ and $M^{\text {gr }}$, by replacing union with sum in the group $\mathbb{Z} \mathcal{E}$.

Definition 2.16 A network in the graded lattice $M^{\mathrm{gr}}$ is a pair $\omega=(1, a) \in M^{\mathrm{gr}}$ where $a \in M$ is a network.

Lemma 2.17 An element of the lattice $M$ is represented by a labeling of the edges of $\mathcal{G}$ with integers so that the sum at any vertex is even. Thus the lattice $M \subset \mathbb{Z} \mathcal{E}$ is generated by

(i) networks

(ii) $\{2 e \mid e \in \mathcal{E}\}$.

Proof Let $\omega \in M$. By using generators of the second type, we can assume that $0 \leq$ $a_{v}(\omega), b_{v}(\omega), c_{v}(\omega) \leq 1$ for any vertex $v$. This implies that $a_{v}(\omega)+b_{v}(\omega)+c_{v}(\omega)$ equals 0 or 2 and either two among $a_{v}(\omega), b_{v}(\omega), c_{v}(\omega)$ are one or all are zero. This implies that $\omega$ is a network, since it corresponds to a disjoint union of paths. A path goes through a vertex $v$ means in terms of $a_{v}(\omega), b_{v}(\omega), c_{v}(\omega)$ that exactly two of them are one.

We define the cone $\tau(\mathcal{G})$ of the graph, which is the semigroup defining the model of the graph as projective spectrum of the semigroup ring, as we will see in Theorem 2.20 .

Definition 2.18 For a graph $\mathcal{G}$ we define its cone $\tau=\tau(\mathcal{G}) \subset M^{\text {gr }}$ as the set of $\omega \in M^{\mathrm{gr}}$ which satisfy following inequalities:

(i) $a_{v}(\omega), b_{v}(\omega), c_{v}(\omega) \geq 0$,

(ii) for any vertex $v \in \mathcal{V}$ triangle inequalities hold

$$
\left|a_{v}(\omega)-b_{v}(\omega)\right| \leq c_{v}(\omega) \leq a_{v}(\omega)+b_{v}(\omega), \quad \text { and }
$$

(iii) $\operatorname{deg}(\omega) \geq \operatorname{deg}_{\min }(\omega)$.

Remark 2.19 To explain the name minimal degree $\operatorname{deg}_{\min }(\omega)$, note that for any $\omega$ in the cone we have the following equality:

$$
\operatorname{deg}_{\min }(\omega)=\min _{\omega^{\prime} \in \tau}\left\{\operatorname{deg}\left(\omega^{\prime}\right): \pi_{\widehat{M}}\left(\omega^{\prime}\right)=\pi_{\widehat{M}}(\omega)\right\}
$$

Proof of the remark By part (iii) of the Definition 2.18 of $\tau$ for any $\omega^{\prime} \in \tau$ satisfying $\pi_{\widehat{M}}\left(\omega^{\prime}\right)=\pi_{\widehat{M}}(\omega)$ we have

$$
\operatorname{deg}\left(\omega^{\prime}\right) \geq \operatorname{deg}_{\min }\left(\omega^{\prime}\right)=\operatorname{deg}_{\min }(\omega)
$$

since by definition of $\operatorname{deg}_{\min }(\omega)$ only depends on $\pi_{\widehat{M}}(\omega)$. This means

$$
\operatorname{deg}_{\min }(\omega) \leq \min _{\omega^{\prime} \in \tau}\left\{\operatorname{deg}\left(\omega^{\prime}\right): \pi_{\widehat{M}}\left(\omega^{\prime}\right)=\pi_{\widehat{M}}(\omega)\right\}
$$


To prove the equality we will find $\omega^{\prime} \in \tau$ with $\operatorname{deg}\left(\omega^{\prime}\right)=\operatorname{deg}_{\min }(\omega)$. Let us write $\omega=(\operatorname{deg}(\omega), \alpha)$ as it is an element of $M^{\text {gr }}$. Recall that $\operatorname{deg}_{\min }(\omega)$ is the maximum of $\operatorname{deg}_{v}(\omega)=\frac{1}{2} \cdot\left(a_{v}+b_{v}+c_{v}\right)(w)$ over all vertices $v$ of the graph. Thus $\omega^{\prime}=\left(\operatorname{deg}_{\min }(\omega), \alpha\right)$ is in the cone $\tau$ and has the required degree in $M^{\mathrm{gr}}$.

Theorem 2.20 The variety $X(\mathcal{G})$ is isomorphic to the toric variety $\operatorname{Proj} \mathbb{C}[\tau(\mathcal{G})]$.

Proof To see this we first observe that each $\mathbb{P}_{v}^{3}$ in Definition 2.11 of the model $X(\mathcal{G})$ can be written as Proj $\mathbb{C}\left[\tau\left(\lambda_{v}\right)\right]$, where each cone

$$
\tau\left(\lambda_{v}\right)=\operatorname{conv}(0000,1000,1110,1101,1011)
$$

is a cone over a tetrahedron and is clearly defined by the required inequalities. Next, taking the product of $\mathbb{P}_{v}$ corresponds by Lemma 1.13 to taking a graded product of cones. Thus the product cone is defined by required inequalities. Lastly we use the description of the quotient of a toric variety by a subtorus of the torus in Theorem 1.14. The subtorus in Definition 2.11 is a product of the $\mathbb{C}_{e}^{*}$ over all internal edges. Taking the quotient with respect to such a torus corresponds to cutting the cone with the hyperplane of the type $a_{\partial_{1}(e)}=b_{\partial_{2}(e)}$, which preserves the inequalities.

\section{$2.4 \mathbb{Z}$-generators of the cone $\tau(\mathcal{G})$}

Knowing that the model $X(\mathcal{G})$ is the projective spectrum of the semigroup algebra of $\tau(\mathcal{G})$ means that it is a subvariety of a weighted projective space with weights equal to the degrees of the chosen generators. When $\mathcal{G}=\mathcal{T}$ is a tree the cone is generated in degree 1 so the embedding is into a (straight) projective space $\mathbb{P}^{k}=\mathbb{P}(1, \ldots, 1)$. In this case, by [3], we already know all about this cone, see Proposition 2.28 below. It is represented by its degree one section-a normal lattice polytope $\Delta(\mathcal{T}) \subset 1 \times M$, whose vertices span the cone $\tau(\mathcal{T})$ and the lattice points generate the semigroup. Our goal is to show that for graphs with the first Betti number one, the semigroup is generated in degrees one and two.

In order to describe $\mathbb{Z}$-generators of the cone $\tau(\mathcal{G})$ we will express elements of $\tau(\mathcal{G})$ in terms of $\mathcal{G}$. We also decompose the graph $\mathcal{G}$ into smaller graphs for which $\mathbb{Z}$-generators of the corresponding cones are easier to find.

We explain that any element $\omega$ of the cone $\tau(\mathcal{G})$ locally decomposes into paths. In the graph $入$ there are three non-empty paths, each consisting of two edges. Let us denote them by

$$
x:=e_{2}+e_{3}, \quad y:=e_{1}+e_{3}, \quad z:=e_{1}+e_{2}
$$

where $\left\{e_{1}, e_{2}, e_{3}\right\}$ are edges of $入$.

For an arbitrary $\mathcal{G}$, we know that given an element $\omega \in \tau(\mathcal{G})$ of the cone and a vertex $v \in \mathcal{V}(\mathcal{G})$, the numbers $a_{v}(\omega), b_{v}(\omega), c_{v}(\omega)$ satisfy the triangle inequalities and their sum is even. This allows us to, locally at $v$, rewrite $\omega$ as sum of paths $x, y$ and $z$. The picture of this decomposition is drawn in Fig. 9.

Our aim is to find the $\mathbb{Z}$-generators by understanding how the graph $\mathcal{G}$ was built from smaller pieces. Each of the operations in Definition 2.5 has a corresponding 
Fig. 9 Local paths around a vertex

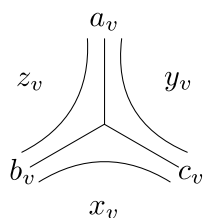

operation on lattices and cones. By Definition 2.11, the model of a disjoint sum of graphs is the product of the models, so the underlying cone is the graded product of corresponding cones.

Lemma 2.21 Let $\mathcal{G}_{1}$ and $\mathcal{G}_{2}$ be two trivalent graphs then

$$
\begin{aligned}
M^{\mathrm{gr}}\left(\mathcal{G}_{1} \sqcup \mathcal{G}_{2}\right) & =M^{\mathrm{gr}}\left(\mathcal{G}_{1}\right) \times_{g} M^{\mathrm{gr}}\left(\mathcal{G}_{2}\right), \quad \text { and } \\
\tau\left(\mathcal{G}_{1} \sqcup \mathcal{G}_{2}\right) & =\tau\left(\mathcal{G}_{1}\right) \times_{g} \tau\left(\mathcal{G}_{2}\right) .
\end{aligned}
$$

In the definition of $X(\mathcal{G})$ we take a quotient by a torus corresponding to the set of inner edges. In other words we have translated the operation of gluing of two leaves of a graph $\mathcal{G}$ into taking a quotient by an appropriate $\mathbb{C}^{*}$-action of the model $X(\mathcal{G})$. The following observation says that if we choose to glue some pairs of leaves first and then the rest of the pairs it does not matter how we partition the set of pairs of leaves or which order we choose. In all cases the resulting variety is the same.

Lemma 2.22 Let two reductive, commutative groups $H_{1}$ and $H_{2}$ and their direct sum $H_{1} \oplus H_{2}$ act on a projective variety $X$. Suppose all those actions are linearized with respect to some ample Weil divisor $\mathcal{L}$. Then

$$
X / /\left(H_{1} \oplus H_{2}\right)=\left(X / / H_{1}\right) / / H_{2}=\left(X / / H_{2}\right) / / H_{1},
$$

where the semistable points on $X$ are taken with respect to $\mathcal{L}$ and on quotients of $X$ with respect to the push-forward of $\mathcal{L}$.

We have identified the vertices of $\mathcal{G}$ with elements of $\mathbb{Z} \mathcal{E}^{\vee}$, see (2.4). We observed in Remark 2.13 that a leaf $l$ yields a $\mathbb{C}^{*}$-action $\lambda^{l}$ on $X(\mathcal{G})$. Given two leaves $l_{1}$ and $l_{2}$ of $\mathcal{G}$, by definition we have

$$
X\left(\mathcal{G}_{l_{2}}^{l_{1}} \supset\right)=X(\mathcal{G}) / /\left(\lambda^{l_{1}} \times-\lambda^{l_{2}}\right) .
$$

In terms of toric geometry this quotient corresponds to the intersection of $\tau(\mathcal{G})$ with the kernel of $l_{1}-l_{2}$, where we treat $l_{1}$ and $l_{2}$ as elements of the lattice $\left(M^{\mathrm{gr}}\right)^{\vee}$. Thus the following lemma is a consequence of Theorem 1.14.

Lemma 2.23 Let $l_{1}$ and $l_{2}$ be distinct leaves of $\mathcal{G}$. Then

$$
\begin{aligned}
M^{\mathrm{gr}}\left(\mathcal{G}_{l_{2}}^{l_{1}} \supset\right) & =M^{\mathrm{gr}}(\mathcal{G}) \cap \operatorname{ker}\left(l_{1}-l_{2}\right), \\
\tau\left(\mathcal{G}_{l_{2}}^{l_{1}} \supset\right) & =\tau(\mathcal{G}) \cap \operatorname{ker}\left(l_{1}-l_{2}\right) .
\end{aligned}
$$


The reverse operation on graphs is to cut an edge into two new edges. In the next lemma we explain how this is reflected on the cones and lattices.

Lemma 2.24 Let $\mathcal{G}$ be a trivalent graph and $e \in \mathcal{E} \backslash \mathcal{P}$ an internal edge. As before $\mathcal{G}^{e}$ is the graph obtained from $\mathcal{G}$ by cutting the edge $e$. Then there are natural embeddings of the cones and graded lattices:

$$
i^{e}: M^{\mathrm{gr}}(\mathcal{G}) \hookrightarrow M^{\mathrm{gr}}\left(\mathcal{G}^{e}\right), \quad i^{e}: \tau(\mathcal{G}) \hookrightarrow \tau\left(\mathcal{G}^{e}\right) .
$$

Proof Let

$$
\mathbb{Z}^{e}(\mathcal{G})=\bigoplus_{e^{\prime} \in \mathcal{E}(\mathcal{G}) \backslash\{e\}} \mathbb{Z} \cdot e^{\prime}
$$

be the lattice spanned by all other edges. We can decompose the lattices $(\mathbb{Z} \mathcal{E})^{\mathrm{gr}}(\mathcal{G})$ and $(\mathbb{Z} \mathcal{E})^{\text {gr }}\left(\mathcal{G}^{e}\right)$

$$
\begin{aligned}
\mathbb{Z} \mathcal{E}(\mathcal{G}) & =\mathbb{Z} \oplus \mathbb{Z} \mathcal{E}^{e}(\mathcal{G}) \oplus \mathbb{Z} \cdot e, \\
\mathbb{Z} \mathcal{E}\left(\mathcal{G}^{e}\right) & =\mathbb{Z} \oplus \mathbb{Z} \mathcal{E}^{e}(\mathcal{G}) \oplus \mathbb{Z} \cdot e_{1} \oplus \mathbb{Z} \cdot e_{2}
\end{aligned}
$$

Thus we can embed the lattices identifying the first two summands and taking a diagonal embedding of the third one: $i^{e}:=\operatorname{id}_{\mathbb{Z}} \oplus \operatorname{id}_{\mathbb{Z} \mathcal{E}^{e}} \oplus \Delta_{\mathbb{Z}}:(\mathbb{Z} \mathcal{E})^{\mathrm{gr}}(\mathcal{G}) \hookrightarrow(\mathbb{Z} \mathcal{E})^{\mathrm{gr}}\left(\mathcal{G}^{e}\right)$. To complete the proof we check that $i^{e}$ restricts to the lattices with parity condition and to the cones.
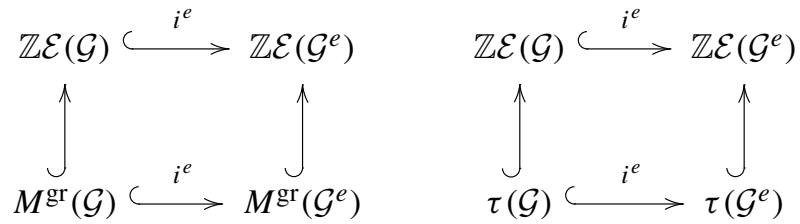

We only need to check the parity condition at the ends $\partial_{1}(e)$ and $\partial_{2}(e)$ of the edge $e$ we cut. By definition $e_{1}^{*}\left(i^{e}(e)\right)=e_{2}^{*}\left(i^{e}(e)\right)$, so for any $\omega \in M^{\text {gr }}$ we have $\partial_{1}(e)(\omega)=$ $\partial_{1}\left(e_{1}\right)\left(i^{e}(\omega)\right)$ and $\partial_{2}(e)(\omega)=\partial_{2}\left(e_{2}\right)\left(i^{e}(\omega)\right)$. In the same way $i^{e}$ preserves all the inequalities defining cones $\tau(\mathcal{G})$ and $\tau\left(\mathcal{G}^{e}\right)$.

When the edge $e$ is not a cycle edge, the graph $\mathcal{G}^{e}$ is not connected. We write $\mathcal{G}^{e}=\mathcal{G}_{1} \sqcup \mathcal{G}_{2}$, where $\mathcal{G}_{1}$ (respectively, $\mathcal{G}_{2}$ ) is the part containing $e_{1}$ (respectively, $e_{2}$ ). Then we have a projection $\pi_{1}^{e}$ (respectively, $\pi_{2}^{e}$ ) of lattices

$$
\pi_{1}^{e}: M^{\mathrm{gr}}\left(\mathcal{G}^{e}\right)=M^{\mathrm{gr}}\left(\mathcal{G}_{1}\right) \times M^{\mathrm{gr}}\left(\mathcal{G}_{2}\right) \rightarrow M^{\mathrm{gr}}\left(\mathcal{G}_{1}\right)
$$

which restricts to a projection of cones. For a non-cycle edge $e \in \mathcal{E}$ we denote by $\rho_{1}^{e}$ (respectively, $\rho_{2}^{e}$ ) the composition $\rho_{1}^{e}=\pi_{1}^{e} \circ i^{e}$ of the above defined maps.

Remark 2.25 When $e \in \mathcal{E}$ is not a cycle edge we write $\mathcal{G}^{e}=\mathcal{G}_{1} \sqcup \mathcal{G}_{2}$. Then the cone $\tau(\mathcal{G})$ is the following fiber product of the cones $\rho_{1}^{e}(\tau(\mathcal{G}))=\tau\left(\mathcal{G}_{1}\right)$ and $\rho_{2}^{e}(\tau(\mathcal{G}))=$ 
$\tau\left(\mathcal{G}_{2}\right)$. The same is true for the lattice $M^{\mathrm{gr}}(\mathcal{G})$.
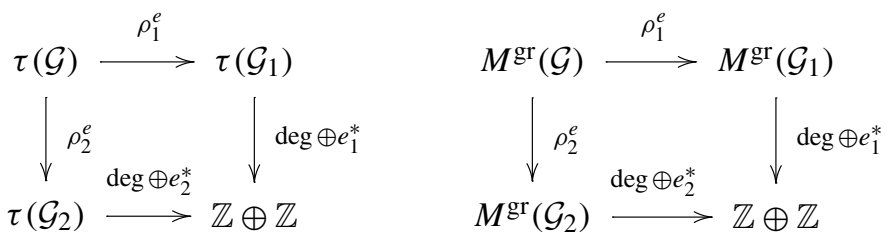

Now we turn our attention to our main task of finding the $\mathbb{Z}$-generators of the cone $\tau(\mathcal{G})$.

Lemma 2.26 For any graph $\mathcal{G}$ the set of degree one integer points of cone $\tau(\mathcal{G})$ is equal to the set of networks.

Proof If $\omega \in \tau$ is a point in the cone of degree one, then for any vertex $v \in \mathcal{V}$,

$$
1=\operatorname{deg}(\omega) \geq \operatorname{deg}_{\min }(\omega)=\max _{u \in \mathcal{V}}\left\{\operatorname{deg}_{u}(\omega)\right\} \geq \operatorname{deg}_{v}(\omega) \geq 0
$$

By definition $\operatorname{deg}_{v}=x_{v}+y_{v}+z_{v} \geq 0$ so exactly one of $x_{v}(\omega), y_{v}(\omega), z_{v}(\omega)$ equals one and the other two are zero or all are zero. Equivalently exactly two of $a_{v}(\omega)$, $b_{v}(\omega), c_{v}(\omega)$ are one, and the third one is zero, or all are zero. This means that $\omega$ is a network.

Corollary 2.27 All networks are among the minimal $\mathbb{Z}$-generators of the cone $\tau(\mathcal{G})$.

In fact when the graph in question is a tree these are the $\mathbb{Z}$-generators.

Proposition $2.28[3, \S 2.1]$ If $\mathcal{G}$ is a trivalent tree, then $\tau(\mathcal{G})$ is generated in degree one. Moreover, the generators are exactly networks of paths, which in this case are determined by their values on the leaves. Thus a generator of $\tau(\mathcal{G})$ is identified with a sequence of 0 's and 1 's of length $n(\mathcal{G})$ with even number of 1 's. As a consequence a model of a trivalent tree with $n$ leaves comes with an embedding into projective space $\mathbb{P}^{2^{n-1}-1}$.

Let $\mathcal{G}$ be a graph with the first Betti number one. We will describe the generators of the semigroup $\tau(\mathcal{G})$ in this case. We cut all the cycle legs $l_{1}, \ldots, l_{k}$ of $\mathcal{G}$ and write $\mathcal{G}^{l_{1}, \ldots, l_{k}}=\mathcal{G}_{0} \sqcup \mathcal{G}_{1} \sqcup \cdots \sqcup \mathcal{G}_{k}$, where $\mathcal{G}_{0}$ is a polygon graph and thus $\mathcal{G}_{1}, \ldots, \mathcal{G}_{k}$ are pendant trees (see Definition 2.6). Thus any element $\omega \in \tau(\mathcal{G})$ has a lift $\tilde{\omega} \in$ $\tau\left(\mathcal{G}_{0} \sqcup \cdots \sqcup \mathcal{G}_{k}\right)=\tau\left(\mathcal{G}_{0}\right) \times{ }_{g} \tau\left(\mathcal{G}_{1}\right) \times{ }_{g} \cdots \times_{g} \tau\left(\mathcal{G}_{k}\right)$ and components $\tilde{\omega}=$ $\left(\omega_{0}, w_{1}, \ldots, \omega_{k}\right)$, which can be written $\omega_{i}=\rho^{l_{i}}(\omega)$.

Theorem 2.29 Let $\mathcal{G}$ be a trivalent graph with the first Betti number exactly one. Any element $\omega \in M^{\mathrm{gr}}(\mathcal{G})$ is a minimal $\mathbb{Z}$-generator of $\tau(\mathcal{G})$ if and only if it satisfies one of the following conditions: 


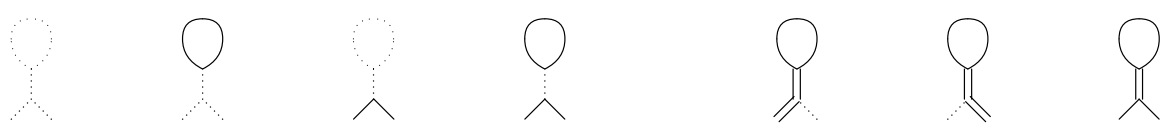

Fig. 10 Generators of the cone of the LittleMan graph

(i) $\omega$ has degree one and $\omega$ is a network, or

(ii) $\omega$ has degree two, and satisfies the following three conditions determining $w_{0}$ :

$$
\begin{aligned}
& e^{*}(\omega)=1, \quad \text { for all cycle edges } e \in \mathcal{E} \backslash \mathcal{L}, \\
& e^{*}(\omega)=2, \quad \text { for an odd number of cycle legs, } \\
& e^{*}(\omega)=0, \quad \text { for the remaining cycle legs. }
\end{aligned}
$$

Each of the remaining components $\omega_{i} \in \tau\left(\mathcal{G}_{i}\right)$ is an element of degree at most two.

We postpone the proof until we prepare for it with some lemmas. The idea of the proof is to use Lemma 2.24 in order to remove the pendant trees $\mathcal{G}_{1}, \ldots, \mathcal{G}_{k}$ and work only with the polygon graph $\mathcal{G}_{0}$. Lemma 2.33 describes all the degree two points of the cone of a polygon graph and distinguishes the generators among them.

Example 2.30 In Fig. 10 we illustrate the generators of the cone associated to the graph LittleMan (one of the two graphs with one cycle and two leaves). The first four are of degree one, the remaining three are of degree two.

Lemma 2.31 (Decomposition propagates to pendant trees) Let $\mathcal{G}$ be any trivalent graph and $\omega \in \tau$ any cone element. Let us also fix a non-cycle edge $e \in \mathcal{E} \backslash \mathcal{P}$ such that $\mathcal{G}^{e}$ is a tree, where $\mathcal{G}^{e}=\mathcal{G}_{1} \sqcup \mathcal{G}_{2}$. Then any decomposition of $\rho_{1}^{e}(\omega)$ lifts to a decomposition of $\omega$.

Proof First note that both $\rho_{1}^{e}$ and $\rho_{2}^{e}$ preserve the degree, so an element $\omega$ of degree $d$ in $\tau(\mathcal{G})$ yields $\omega_{1} \in \tau\left(\mathcal{G}_{1}\right)$ and $\omega_{2} \in \tau\left(\mathcal{G}_{2}\right)$ both of degree $d$. The semigroup of a tree is generated by networks, which are degree one elements, see Theorem 2.28 . This means that $\rho_{2}^{e}(\omega)$ is a sum of degree one elements. Thus if $\rho_{1}^{e}(\omega)$ can be decomposed, then the same decomposition works for $\omega$ by choosing an appropriate grouping of the summands of $\rho_{2}^{e}(\omega)$, because the degrees are preserved.

Corollary 2.32 In the proof of Theorem 2.29 we can assume that the graph is a polygon graph.

Proof A graph with one cycle is a polygon graph with a tree attached to each cycle leg $l_{1}, \ldots, l_{k}$. We cut all the cycle legs to obtain $k+1$ pieces of the graph $\mathcal{G}$ : a polygon graph $\mathcal{G}_{0}$ and $k$ trees: $\mathcal{G}_{1}, \ldots, \mathcal{G}_{k}$. We denote by $\rho_{0}$ the composition of projections for each leg that we cut $\rho_{0}=\rho_{0}^{l_{1}} \circ \cdots \circ \rho_{0}^{l_{k}}$. Iteratively using Lemma 2.31 to decompose $\rho_{0}(\omega)$ we decompose $\omega$. 
Fig. 11 Decomposition of a degree two element with a weight zero cycle edge

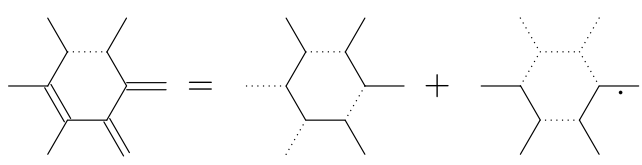

Fig. 12 Decomposition of a degree two element with all cycle edges of weight two

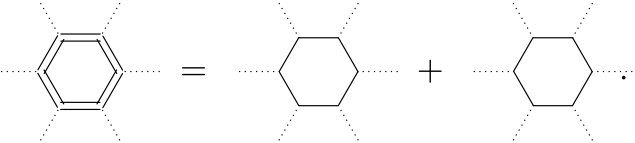

Lemma 2.33 (Degree two elements of the semigroup) Let $\mathcal{G}$ be a graph with exactly one cycle. Any degree two element of $\tau(\mathcal{G})$ except those in Theorem 2.29(ii) is a sum of two networks.

Proof By Corollary 2.32 we may assume $\mathcal{G}$ is a polygon graph. Let $\omega \in \tau(\mathcal{G})$ be a degree two element. The coefficient $e^{*}(\omega)$ of $\omega$ on any edge $e \in \mathcal{E}$ is 0,1 or 2 . We denote by

$$
\omega_{\mathcal{E} \backslash \mathcal{P}}:=\left\{e^{*}(\omega) \mid e \in \mathcal{E} \backslash \mathcal{P}\right\}
$$

the set of coefficients of $\omega$ on the cycle edges. We distinguish between four types of

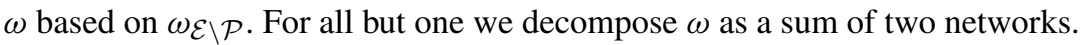

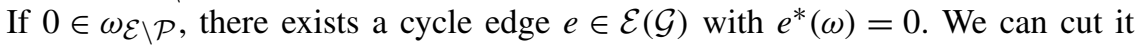
with no harm to $\omega$, since $i^{e}(\omega) \in \tau\left(\mathcal{G}^{e}\right)$ is a degree two element in a cone of the trivalent tree $\mathcal{G}^{e}$, so it can be decomposed into a sum of degree one elements. This decomposition can be lifted to $\tau(\mathcal{G})$, as we assumed $e^{*}(\omega)=0$. In Fig. 11 we show an example of this situation.

The second case is $\omega_{\mathcal{E} \backslash \mathcal{P}}=\{2\}$, that is, $e^{*}(\omega)$ is 2 on all cycle edges. As $\omega$ has degree two, we know that $\operatorname{deg}_{v}(\omega) \leq 2$ and as a consequence

$$
e^{*}(\omega)= \begin{cases}2, & \text { if } e \text { is a cycle edge } \\ 0, & \text { otherwise, i.e. } e \text { is a cycle leg. }\end{cases}
$$

Thus $\omega$ is twice the network consisting of all the cycle edges, as in the example in Fig. 12.

For the last two cases we let $l_{1}, \ldots, l_{p}$ be the set of all cycle legs with $l_{i}^{*}(\omega) \neq 0$ ordered anticlockwise and indexed by elements of $\mathbb{Z}_{p}$. Also we denote by $i \curvearrowleft j$ the path that starts at $l_{i}$ and goes anticlockwise along the intermediate cycle edges to $l_{j}$ and ends there.

In the third case $\omega_{\mathcal{E} \backslash \mathcal{P}}=\{1\}$ and we will show that $\omega$ can be decomposed into a sum of two networks if and only if the number of cycle legs for which $\omega$ has a coefficient 2 is even. Suppose we have decomposed $\omega=\omega_{1}+\omega_{2}$ into a sum of networks and neither $\omega_{1}$ nor $\omega_{2}$ contains the path consisting of all cycle edges (in which case the other one would be an empty network). Then both $\omega_{1}$ and $\omega_{2}$ contain a positive even number of legs. Any path in $\omega_{1}$ (respectively, $\omega_{2}$ ) is of the type $i \curvearrowleft i+1$, from the cycle leg $l_{i}$ to the next one. If the end was not the next one, there would be a path in $\omega_{2}$ (respectively, $\omega_{1}$ ) containing an intermediate leg and as a result there would be 

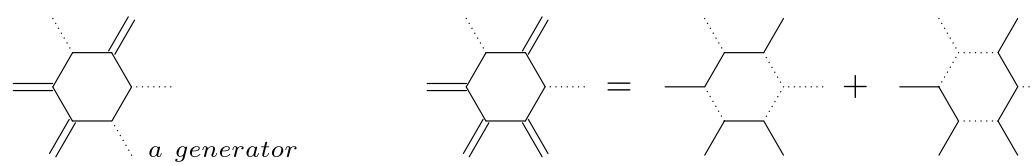

Fig. 13 Typical generator of degree two and a decomposition of a degree two element with all cycle edges of weight one

Fig. 14 Element of degree two has even number of one-legs between consecutive two-legs
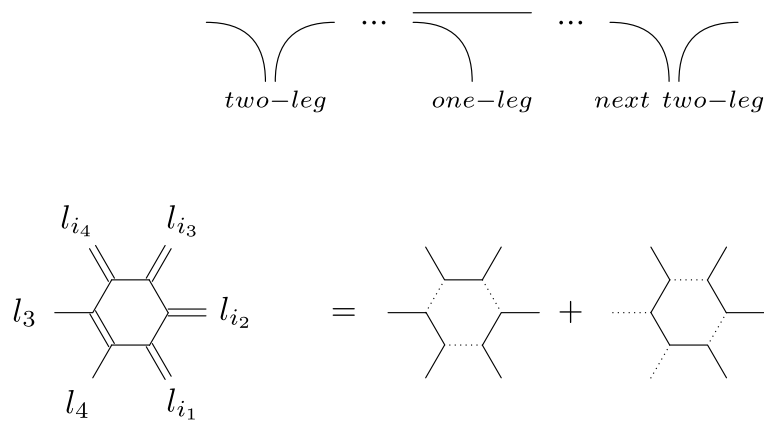

Fig. 15 Decomposition of a degree two element with even number of two-legs

a cycle edge $e$ with $\omega(e)=2$, but $\omega_{\mathcal{E} \backslash \mathcal{P}}=\{1\}$. Moreover, both $\omega_{1}$ and $\omega_{2}$ contain all non-zero cycle legs, each with value 1 , because they are both of degree 1 and in the cone we have $\operatorname{deg}(\omega) \geq \operatorname{deg}_{\min }(\omega)$. This is only possible when the number $p$ of non-zero cycle legs is even, and in that case we have the obvious decomposition with $\omega_{1}=i_{1} \curvearrowleft i_{2}+\cdots+i_{p-1} \curvearrowleft i_{p}$ and $\omega_{2}=i_{2} \curvearrowleft i_{3}+\cdots+i_{p} \curvearrowleft i_{1}$. Otherwise $\omega$ is a generator. Examples both of these situations are drawn in Fig. 13.

In the last case $\omega_{\mathcal{E} \backslash \mathcal{P}}=\{1,2\}$. When $l^{*}(\omega)=2$ we call $l$ a two-leg. Denote by $l_{i_{1}}, \ldots, l_{i_{q}}$ the subsequence of two-legs, numbered in such way that traveling along the cycle anticlockwise from $l_{i_{q}}$ to $l_{i_{1}}$ there is a one-leg, provided that $\omega$ has some one-legs. We observe that the number of one-legs between two consecutive two-legs is always even. This is best explained by drawing the picture from Fig. 14, where the arcs are our $x_{v}, y_{v}, z_{v}$ 's introduced in Fig. 9. If there would be only zero-legs where the first dots are, the local paths would not agree on some cycle edge.

The decomposition $\omega=\left(1, \omega_{1}\right)+\left(1, \omega_{2}\right)$ depends on the parity of $q$, which is the number of two-legs. We first work out the case when $q=2 r$ is even; the odd case uses the same idea with small modifications. Figure 15 visualizes how the decomposition is constructed in the case of $q=4$.

First we place all paths connecting two consecutive two-legs starting at a leg with an even (respectively, odd) index into $\omega_{1}$ (respectively, $\omega_{2}$ ). Then, to take care of the one-legs, we add paths between consecutive one-legs lying between $l_{i_{2 j}}$ and $l_{i_{2 j+1}}$ for some $j \in\{1, \ldots, p\}$ to $\omega_{1}$. Thus we get

$$
\begin{aligned}
\omega_{1}= & i_{1} \curvearrowleft i_{2}+\left(i_{2}+1\right) \curvearrowleft\left(i_{2}+2\right)+\cdots+\left(i_{3}-2\right) \curvearrowleft\left(i_{3}-1\right) \\
& +i_{3} \curvearrowleft i_{4}+\left(i_{4}+1\right) \curvearrowleft\left(i_{4}+2\right)+\cdots+\left(i_{5}-2\right) \curvearrowleft\left(i_{5}-1\right) \\
& +\cdots \\
& +i_{2 r-1} \curvearrowleft i_{2 r}+\left(i_{2 r}+1\right) \curvearrowleft\left(i_{2 r}+2\right)+\cdots+\left(i_{1}-2\right) \curvearrowleft\left(i_{1}-1\right) .
\end{aligned}
$$


Fig. 16 Decomposition of a degree two element with odd number of two-legs
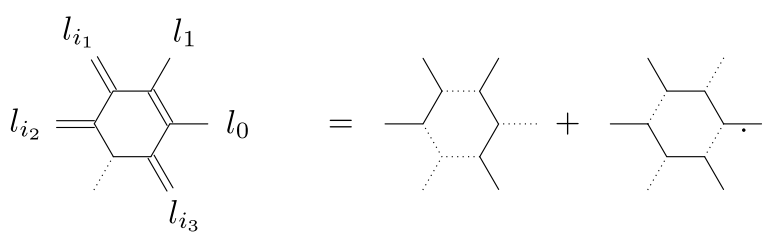

Similarly we add paths between consecutive one-legs lying between $l_{i_{2 j-1}}$ and $l_{i_{2 j}}$ for some $j \in\{1, \ldots, p\}$ to $\omega_{2}$. So we can write

$$
\begin{aligned}
\omega_{2}= & i_{2} \curvearrowleft i_{3}+\left(i_{3}+1\right) \curvearrowleft\left(i_{3}+2\right)+\cdots+\left(i_{4}-2\right) \curvearrowleft\left(i_{4}-1\right) \\
& +i_{4} \curvearrowleft i_{5}+\left(i_{5}+1\right) \curvearrowleft\left(i_{5}+2\right)+\cdots+\left(i_{6}-2\right) \curvearrowleft\left(i_{6}-1\right) \\
& +\cdots \\
& +i_{2 r} \curvearrowleft i_{1}+\left(i_{1}+1\right) \curvearrowleft\left(i_{1}+2\right)+\cdots+\left(i_{2}-2\right) \curvearrowleft\left(i_{2}-1\right) .
\end{aligned}
$$

Clearly those paths in $\omega_{1}$ (resp. $\omega_{2}$ ) are disjoint, so both are networks and by construction they yield a decomposition of our $\omega$.

When the number $q$ of two-legs is odd we need to adjust the above decomposition. Again, we draw an example in Fig. 16 for $q=3$.

As there is an odd number of two-legs, we will use two consecutive one-legs lying between $l_{i_{q}}$ and $l_{i_{1}}$ to make up for the missing two-leg, and we proceed as before to get

$$
\begin{aligned}
\omega_{1}= & i_{1} \curvearrowleft i_{2}+\left(i_{2}+1\right) \curvearrowleft\left(i_{2}+2\right)+\cdots+\left(i_{3}-2\right) \curvearrowleft\left(i_{3}-1\right) \\
& +i_{3} \curvearrowleft i_{4}+\left(i_{4}+1\right) \curvearrowleft\left(i_{4}+2\right)+\cdots+\left(i_{5}-2\right) \curvearrowleft\left(i_{5}-1\right) \\
& +\cdots \\
& +i_{2 r-1} \curvearrowleft i_{2 r}+\left(i_{2 r}+1\right) \curvearrowleft\left(i_{2 r}+2\right)+\cdots+\left(i_{2 r+1}-2\right) \curvearrowleft\left(i_{2 r+1}-1\right) \\
& +i_{2 r+1} \curvearrowleft i_{1}-1
\end{aligned}
$$

and

$$
\begin{aligned}
\omega_{2}= & i_{2} \curvearrowleft i_{3}+\left(i_{3}+1\right) \curvearrowleft\left(i_{3}+2\right)+\cdots+\left(i_{4}-2\right) \curvearrowleft\left(i_{4}-1\right) \\
& +i_{4} \curvearrowleft i_{5}+\left(i_{5}+1\right) \curvearrowleft\left(i_{5}+2\right)+\cdots+\left(i_{6}-2\right) \curvearrowleft\left(i_{6}-1\right) \\
& +\cdots \\
& +i_{2 r} \curvearrowleft i_{2 r+1}+\left(i_{2 r+1}+1\right) \curvearrowleft\left(i_{2 r+1}+2\right)+\cdots+\left(i_{1}-4\right) \curvearrowleft\left(i_{1}-3\right) \\
& +i_{1}-2 \curvearrowleft i_{1} .
\end{aligned}
$$

This ends the proof of the lemma about the decomposable degree two elements of the cone.

Proof of Theorem 2.29 The proof yields an algorithm for decomposing an arbitrary element $\omega$ of the cone $\tau(\mathcal{G})$ into a sum of degree one and two generators. By Corollary 2.32 we only need to prove the theorem when $\mathcal{G}$ is a polygon graph. First we fix an orientation of the cycle of $\mathcal{G}$ and we call it anticlockwise in order to think of a planar embedding of the graph. Let $\omega \in \tau(\mathcal{G})$. We will find an element $\mu$ of degree at most two, such that $\omega-\mu \in \tau(\mathcal{G})$. Let $v$ be a vertex and $l_{v}$ the cycle leg attached 
to it. We choose a map $i_{v}: \curlywedge \rightarrow \mathcal{G}$ so that the edge $e_{3}$ is mapped to $l_{v}$, the edge $e_{2}$ is mapped to the edge which points anticlockwise from $v$, and the edge $e_{1}$ is mapped to the edge which points clockwise from $v$. In this notation the coefficient of $\omega$ at $l_{v}$ is measured by $c_{v}$ and the coefficient of the edge anticlockwise (respectively, clockwise) from $v$ is measured by $a_{v}$ (respectively, $b_{v}$ ). We will also use the local paths $x_{v}, y_{v}, z_{v}$ defined in Fig. 9.

Now we are ready to describe the algorithm to find $\mu$.

Step 1. If there is a cycle edge $e$ with $e^{*}(\omega)=0$, we cut $e$ and obtain the graph $\mathcal{G}^{e}$ which is a trivalent tree. Thus $\rho^{e}(\omega)$ is a sum of networks of paths and this decomposition lifts to graph $\mathcal{G}$.

Step 2. Otherwise $e^{*}(\omega) \geq 1$ on all cycle edges $e$. We set $\mu$ to have value 1 on every cycle edge. Equivalently $\mu$ is defined by setting at every cycle vertex $z_{v}(\mu)=1$, $x_{v}(\mu)=0, y_{v}(\mu)=0$. Now if $\omega-\mu \in \tau(\mathcal{G})$ we are done. Otherwise $\omega-\mu$ fails one of the inequalities defining $\tau(\mathcal{G})$. It is not the one with degrees, since for each vertex $v \in \mathcal{G}$ we have $\operatorname{deg}_{v}(\mu)=1$, which implies $\operatorname{deg}_{\min }(\omega-\mu) \leq \operatorname{deg}(\omega)-\operatorname{deg}(\mu)=$ $\operatorname{deg}(\omega-\mu)$. Clearly all coefficients of $\omega-\mu$ are positive. Thus at some vertex $v \in \mathcal{V}$ our $\omega-\mu$ fails one of the triangle inequalities.

Step 3. We will adjust $\mu$ to fix the triangle inequalities for $\omega-\mu$. If a triangle inequality for $\omega-\mu$ at $v$ fails, then this is because $z_{v}(\omega)=0$. In such a case we set $\mu\left(l_{v}\right)=2$, which will not make any coefficient of $\omega-\mu$ negative provided $c_{v}(\omega) \geq 2$. But since $a_{v}(\omega), b_{v}(\omega) \geq 1$ and $z_{v}(\omega)=0$ we must have $x_{v}(\omega)$, $y_{v}(\omega) \geq 1$. This implies $c_{v}(\omega) \geq 2$ as required. In terms of $x_{v}, y_{v}, z_{v}$ we have decreased $z_{v}(\mu)$ by one and increased both $x_{v}(\mu)$ and $y_{v}(\mu)$ by one.

Step 4. The last step adjusts $\mu$ to ensure the additivity of degree where it is attained, i.e. for any $v$ such that $\operatorname{deg}_{v}(\omega)=\operatorname{deg}(\omega)$ we need $\operatorname{deg}_{v}(\mu)=2$ since $\operatorname{deg}(\mu)=2$. This is to ensure $\omega-\mu \in \tau$. We call $v$ degree deficient vertex if $\operatorname{deg}_{v}(\omega)=\operatorname{deg}(\omega)$ and $\operatorname{deg}_{v}(\mu)=1$.

If $v$ is degree deficient and in addition $x_{v}(\omega)>0$ and $y_{v}(\omega)>0$ we set $x_{v}(\mu)=$ $y_{v}(\mu)=1$ and $z_{v}(\mu)=0$.

If $v$ is degree deficient and both $x_{v}(\omega)=y_{v}(\omega)=0$ are zero, then $z_{v}(\omega)=\operatorname{deg}_{v}(\omega)$, so at both next and previous cycle vertex the degree is attained

$$
\operatorname{deg}_{v_{\text {next }}}(\omega)=\operatorname{deg}_{v_{\text {prev }}}(\omega)=\operatorname{deg}(\omega)
$$

since path matching gives

$$
\begin{aligned}
z_{v_{\text {next }}}+y_{v_{\text {next }}} & =z_{v}+x_{v}=\operatorname{deg}(\omega)=z_{v}+y_{v} \\
& =z_{v_{\text {prev }}}+x_{v_{\text {prev }}} .
\end{aligned}
$$

If all degree deficient vertices were of this type, then $\omega$ would be a multiple of the path consisting of all cycle edges.

Now we divide the set of all deficient vertices (which all have at least one of $x_{v}(\omega)$ or $y_{v}(\omega)$ equal to zero) into sequences of adjacent ones. Let us fix our attention to such a sequence (we have already excluded the case when it has the same end and beginning). Call it $v_{1}, \ldots, v_{r}$. All $v_{i}$ 's are degree deficient so $\operatorname{deg}(\omega)=\operatorname{deg}_{v_{i}}(\omega)$. 
Fig. 17 Example of a degree three generator

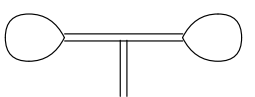

The last one has $y_{v_{r}}(\omega)>0$ by $(2.5)$

$$
\operatorname{deg}(\omega)=z_{v_{r-1}}(\omega)=y_{v_{r}}(\omega)+z_{v_{r}}(\omega) \leq z_{v_{r}}(\omega)<\operatorname{deg}(\omega) .
$$

In the same way $x_{v_{1}}(\omega)>0$. Finally all the middle ones have $z_{v_{i}}(\omega)>1$. This implies that we can redefine $\mu$ on our fixed sequence $v_{1}, \ldots, v_{r}$ preserving all other properties and fixing the degree deficiency:

$$
\begin{array}{lll}
x_{v_{1}}(\mu)=1, & x_{v_{i}}(\mu)=0, & x_{v_{r}}(\mu)=0, \\
y_{v_{1}}(\mu)=0, & y_{v_{i}}(\mu)=0, & y_{v_{r}}(\mu)=1, \\
z_{v_{1}}(\mu)=1, & z_{v_{i}}(\mu)=2, & z_{v_{r}}(\mu)=1
\end{array}
$$

where $i \in\{2, \ldots, r-1\}$. We do this for all such sequences and we have the required $\mu$.

Now $\mu$ is an element of degree two and is either a generator or can be decomposed into a sum of two generators in degree one, as described in Lemma 2.33.

Remark 2.34 If we allow more cycles, we can have generators of higher degree. As we can see in Fig. 17 the graph with two loops and one leaf has a degree three generator: one on the two loops, two on the three other edges.

\subsection{Embedding}

The aim of this section is to find a common ambient space for phylogenetic models of all graphs with the same topological invariants. The way to construct this ambient space follows easily form the definition of the model.

Theorem 2.35 The phylogenetic model of a trivalent graph $\mathcal{G}$ embeds in a projective toric variety $\mathbb{P}_{g, n}$, which is a good quotient of projective space by an action of a $g$ dimensional torus. This action as well as the variety $\mathbb{P}_{g, n}$ depends only on the first Betti number and number of leaves of $\mathcal{G}$, up to reordering of coordinates.

Proof Models of trivalent trees with $N$ leaves embed naturally in $\mathbb{P}^{2^{N-1}-1}$ with coordinates $x_{\kappa}$ where $\kappa \in\{0,1\}^{N}$ is a sequence of length $N$ with values in $\{0,1\}$ and even number of 1-entries, see Proposition 2.28. We cut $g$ cycle edges of the graph $\mathcal{G}$ so we obtain a trivalent tree $\mathcal{T}$ with set $S$ of $N=n+2 g$ leaves. Dividing $\mathbb{P}^{2^{N-1}-1}$ by the action of the $g$-dimensional torus $\mathbb{T}(g, n)$ corresponding to gluing these leaves back together yields the required embeddings.

We will now explain why this action does not depend on the graph $\mathcal{G}$, up to choice of coordinates on $\mathbb{P}^{2^{N}-1}$. Let us label the set of leaves by $1, \ldots, N$ and divide it into three disjoint sets $S=S_{0} \sqcup S_{1} \sqcup S_{-1}$ as follows. $S_{0}$ is the set of leaves of the original graph $\mathcal{G}$. The $2 g$ new leaves of $\mathcal{T}$ come in pairs $\left(l, l^{-}\right)$, where both $l$ and $l^{-}$used to be the same edge in $\mathcal{G}$. We put $l$ in $S_{1}$ and $l^{-}$in $S_{-1}$. 
Fig. 18 Obtaining LittleMan graph from a four-leaf tree

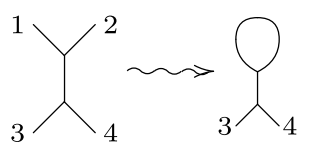

The action of the $g$-dimensional torus $\mathbb{T}(g, n)$ on $\mathbb{P}^{2^{N-1}-1}$ is given by a matrix

$$
\left\{\zeta_{\kappa}^{l}\right\}_{\kappa \in\{0,1\}^{N}}^{l=1 \ldots g}, \quad \text { where } \zeta_{\kappa}^{l}=\kappa_{l}-\kappa_{l^{-}} .
$$

Thus this action is independent of the shape of $\mathcal{G}$ and depends only on $(g, n)$ up to choice of order of coordinates.

We will now illustrate Theorem 2.35.

Example 2.36 We describe $X(\mathcal{X})$, the model of LittleMan, together with its embedding into $\mathbb{P}_{1,2}$. We know that the model of a trivalent tree $Y$ is a complete intersection of two quadrics, see [3, Example 2.6],

$$
\begin{aligned}
& \left(x_{1100} x_{0011}-x_{0000} x_{1111}, \quad x_{1001} x_{0110}-x_{1010} x_{0101}\right) \\
& \quad \subset \operatorname{Proj} \mathbb{C}\left[x_{0000}, x_{1100}, x_{0011}, x_{1111}, x_{1010}, x_{1001}, x_{0101}, x_{0110}\right]
\end{aligned}
$$

where each variable $x_{\kappa}$ corresponds to a degree one generator $\tau(Y)$. By Proposition 2.28 a generator is given by its values on the leaves, which form the index $\kappa \in\{0,1\}^{4}$.

To obtain $X(9)$, we glue two leaves, say leaf $e_{1}$ is glued with the leaf $e_{2}$ to get the loop in LittleMan as shown in Fig. 18.

Now $X(\mathcal{X})$ is a GIT-quotient of $X(Y)$ by the action $\lambda^{e_{1}-e_{2}}$.

In the same way it embeds in a GIT-quotient of $\mathbb{P}^{7}$. One easily sees that

$$
\mathbb{P}^{7} / / \mathbb{C}^{*}=V\left(y_{1} \cdot y_{2}-z_{1} \cdot z_{2}\right) \subset \mathbb{P}\left(1^{4}, 2^{4}\right)
$$

where

$$
\mathbb{P}\left(1^{4}, 2^{4}\right)=\operatorname{Proj} \mathbb{C}\left[x_{0000}, x_{1100}, x_{0011}, x_{1111}, y_{1}, y_{2}, z_{1}, z_{2}\right]
$$

is a weighted projective space and

$$
\begin{array}{ll}
y_{1}=x_{1001} \cdot x_{0110}, & y_{2}=x_{1010} \cdot x_{0101}, \\
z_{1}=x_{1010} \cdot x_{0110}, & z_{2}=x_{0101} \cdot x_{1001}
\end{array}
$$

are the $\mathbb{C}^{*}$-invariant variables of degree two. From Theorem 1.6 it follows that $X(9)$ is given by (2.6) of $X(Y)$ in $\mathbb{P}^{7}$, rewritten in the coordinates of $\mathbb{P}^{7} / / \mathbb{C}^{*}$. The second one becomes $y_{1}-y_{2}=0$, so

$$
\begin{aligned}
& X(\mathcal{Q})=\operatorname{Proj} \mathbb{C}[\tau(9)]=V\left(x_{1100} x_{0011}-x_{0000} x_{1111}, y_{1}^{2}-z_{1} z_{2}\right) \subset \mathbb{P}\left(1^{4}, 2^{3}\right) \\
& \bigcap \\
& V\left(y_{1}-y_{2}\right) \\
& \text { १ } \\
& \mathbb{P}_{1,2}=\mathbb{P}^{7} / / \mathbb{C}^{*}=\quad V\left(y_{1} y_{2}-z_{1} z_{2}\right) \quad \subset \mathbb{P}\left(1^{4}, 2^{4}\right)
\end{aligned}
$$




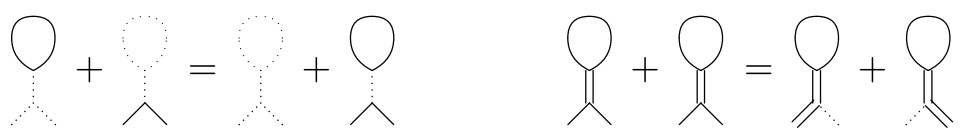

Fig. 19 Ideal of the model LittleMan graph

Fig. 20 Obtaining Hammock graph from a four-leaf tree

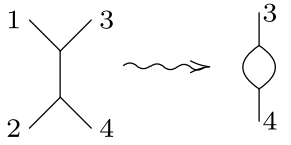

Fig. 21 Ideal of the model of the Hammock graph

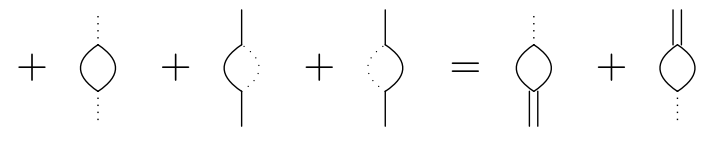

If we replace each variable in the equations of $X(\mathcal{\Lambda})$ by its representation on the graph, we get the picture from Fig. 19.

Example 2.37 To complete the example of Theorem 2.35 for graphs with $g=1$ and $n=2$, which models embed in $\mathbb{P}_{1,2}$, we will find the equations of $X(--)$ - the model of Hammock. We use the same action $\lambda^{l_{1}-l_{2}}$, but we change the embedding $X(Y) \hookrightarrow$ $\mathbb{P}^{7}$ by relabeling the leaves as in Fig. 20.

As the labeling of $Y$ was modified, (2.6) becomes

$$
V\left(x_{1010} x_{0101}-x_{0000} x_{1111}, x_{1001} x_{0110}-x_{1100} x_{0011}\right) \subset \mathbb{P}^{7} .
$$

As before rewrite it in the invariant coordinates of $\mathbb{P}\left(1^{4}, 2^{4}\right)$ to get

$$
\begin{aligned}
& X(-)=\operatorname{Proj} \mathbb{C}[\tau(-\circ)]=V\left(x_{0000} x_{1111} x_{1100} x_{0011}-z_{1} z_{2}\right) \subset \mathbb{P}\left(1^{4}, 2^{2}\right) \\
& V\left(y_{1}-x_{0000} x_{1111}, x_{1100} x_{0011}-y_{2}\right) \\
& \mathbb{P}_{1,2}=\quad \mathbb{P}^{7} / / \mathbb{C}^{*} \quad=\quad V\left(y_{1} y_{2}-z_{1} z_{2}\right) \\
& \subset \mathbb{P}\left(1^{4}, 2^{4}\right) \text {. }
\end{aligned}
$$

If we replace each variable in the degree four equation of $X(-\odot)$ by its representation on the graph, we get the picture shown in Fig. 21.

\section{Flat families}

Models of trivalent trees that differ by one mutation live in a flat family in a projective space [3]. This statement almost remains true for trivalent graphs, by the same argument. The only difference is that we get a family in the projective toric variety $\mathbb{P}_{g, n}$ instead of a usual projective space.

Recall that in Remark 2.13 we have associated to a subset of leaves $\mathcal{S} \subset \mathcal{L}$ with $k$ elements an action of the $k$-dimensional torus $\mathbb{T}(\mathcal{S})$ on the model $X(\mathcal{G})$. 

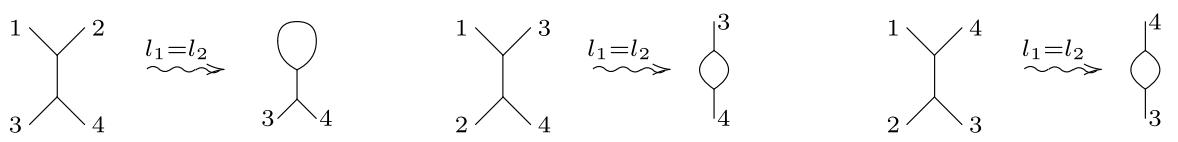

Fig. 22 Mutations of graphs with one cycle and two leaves

\subsection{Key examples}

We construct two-dimensional flat families containing models of small graphs. They become the building blocks for deformations of bigger graphs.

Example 3.1 $(g=0, n=4)$ Let $Y$ be a trivalent tree with four leaves. In [3, Example 2.20] we constructed a flat family

$$
\mathcal{X}^{0} \hookrightarrow \mathcal{B} \times \mathbb{P}^{7},
$$

where

- $\mathcal{B}$ is an open subset of $\mathbb{P}^{2}$ with coordinates $b_{(1,2)(3,4)}, b_{(1,3)(2,4)}, b_{(1,4)(2,3)}$,

- the torus $\mathbb{T}(\mathcal{L})$ acts on $\mathcal{B} \times \mathbb{P}^{7}$ via the second coordinate, that is, for a leaf $l$ of $Y$ and coordinate $x_{\kappa}$ we have $\lambda_{v_{i}}(t)\left(b_{(.)(.)}, x_{\kappa}\right)=\left(b_{(.)(.)}, t^{\kappa(l)} x_{\kappa}\right)$,

- the equidimensional projection $\mathcal{X}^{0} \rightarrow \mathcal{B}$ contains the three special fibers $\mathcal{X}_{[1,0,0]}^{0}$, $\mathcal{X}_{[0,1,0]}^{0}$ and $\mathcal{X}_{[0,0,1]}^{0}$ which are models of aforementioned four-leaf trees,

- $\mathcal{X}^{0}$ is a $\mathbb{T}(\mathcal{L})$-invariant complete intersection in $\mathcal{B} \times \mathbb{P}^{7}$ of the two quadrics

$$
\begin{aligned}
& b_{(12)(34)} \cdot x_{1100} x_{0011}+b_{(13)(24)} \cdot x_{1010} x_{0101}+b_{(14)(23)} \cdot x_{1001} x_{0110} \\
& \quad-\left(b_{(12)(34)}+b_{(13)(24)}+b_{(14)(23)}\right) \cdot x_{0000} x_{1111} \\
& \left(b_{(13)(24)}-b_{(14)(23)}\right) \cdot x_{1100} x_{0011}+\left(b_{(14)(23)}-b_{(12)(34)}\right) \cdot x_{1010} x_{0101} \\
& \quad+\left(b_{(12)(34)}-b_{(13)(24)}\right) \cdot x_{1001} x_{0110} .
\end{aligned}
$$

Example 3.2 ( $g=1, n=2)$ We construct a family which contains models of graphs with one cycle and two leaves. It arises as a $\mathbb{C}^{*}$-quotient of the family $\mathcal{X}^{0} \hookrightarrow \mathbb{P}^{7}$ from Example 3.1. Let us fix a $\mathbb{C}^{*}$-action $\lambda^{l_{1}-l_{2}}$ on the ambient $\mathbb{P}^{7}$, thus on $\mathcal{X}^{0}$ and tree models as well, by choosing leaves $l_{1}$ and $l_{2}$ labeled by 1 and 2 , respectively. Each of the three trees yields a graph, when two leaves are glued together. Up to graph isomorphism, there are two graphs with one cycle and two leaves. As we are mutating along fixed edge, the LittleMan appears once and the Hammock twice. The picture of the three possible trees becomes the one in Fig. 22.

The new ambient space $\mathbb{P}^{7} / / \mathbb{C}^{*}=\left(y_{1} \cdot y_{2}-z_{1} \cdot z_{2}\right) \subset \mathbb{P}\left(1^{4}, 2^{4}\right)$ was discussed in Example 2.36.

By Theorem 1.6, the new family $\mathcal{X}^{0} / / \mathbb{C}^{*}$ is given by the same, $\mathbb{T}(S)$-invariant equations of $\mathcal{X}^{0}$. We rewrite them in the (invariant) coordinates of $\mathbb{P}\left(1^{4}, 2^{4}\right)$

$$
\begin{gathered}
b_{(12)(34)} \cdot x_{1100} x_{0011}+b_{(13)(24)} \cdot y_{2}+b_{(14)(23)} \cdot y_{1} \\
-\left(b_{(12)(23)}+b_{(13)(24)}+b_{(14)(23)}\right) \cdot x_{0000} x_{1111},
\end{gathered}
$$



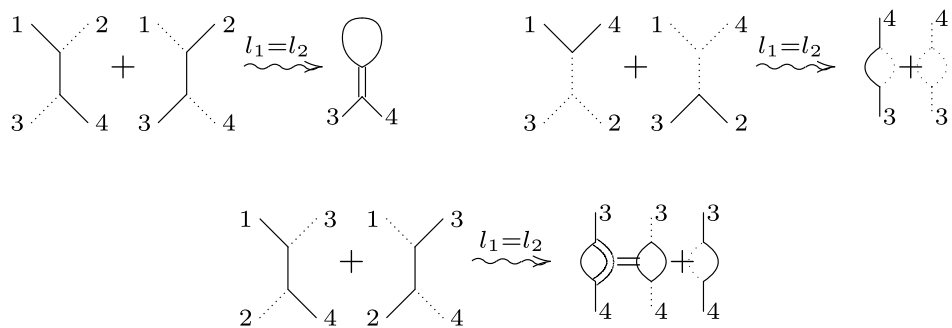

Fig. 23 Behavior of coordinates of $\mathbb{P}_{1,2}$ under mutations

$$
\begin{aligned}
& \left(b_{(23)(14)}-b_{(14)(23)}\right) \cdot x_{1100} x_{0011}+\left(b_{(14)(23)}-b_{(12)(34)}\right) \cdot y_{2} \\
& \quad+\left(b_{(12)(34)}-b_{(23)(14)}\right) \cdot y_{1}, \\
& y_{1} \cdot y_{2}-z_{1} \cdot z_{2} .
\end{aligned}
$$

To understand how this works a little better, let us look at particular coordinate of $\mathbb{P}\left(1^{4}, 2^{4}\right)$, say $y_{2}=x_{1001} x_{0110}$, and draw in Fig. 23 its representation for each graph.

\subsection{General case}

We construct a flat family containing models of all mutations of a given trivalent graph $\mathcal{G}$ along a fixed edge $e \in \mathcal{E} \backslash \mathcal{P}$. We follow [3, Const. 3.5] replacing polytopes by cones.

Let $\mathcal{G}$ be a graph with an inner edge $e_{0}$ which contains two trivalent inner vertices. We can write $\mathcal{G}$ as a sum of a not necessarily connected graph $\mathcal{G}_{1}$ with a set $S$ of $k$ distinguished leaves $l_{1}, \ldots, l_{k}$ with $k \in\{0, \ldots, 4\}$ and a graph $\mathcal{G}_{0}$ having the edge $e_{0}$ as its inner edge and $\mathcal{G}_{0}$ is one of the following three small graphs:

(i) a tree $Y$ with four leaves $v_{1}, \ldots, v_{4}$, where the edge incident to the leaf $l_{i}$ is identified with the edge incident to the leaf $v_{i}$,

(ii) LittleMan 9 or Hammock $\_-$a graph with four edges and two leaves $v_{1}$ and $v_{2}$,

(iii) Dumbell $\&$ a graph with three edges and two loops and no leaves.

From Proposition 2.21 we can compute the lattice $M^{\mathrm{gr}}(\mathcal{G})$ and the cone $\tau(\mathcal{G})$ from those of the pieces $\mathcal{G}_{0}$ and $\mathcal{G}_{1}$

$$
\begin{gathered}
M^{\mathrm{gr}}(\mathcal{G})=M^{\mathrm{gr}}\left(\mathcal{G}_{0}\right) \times{ }_{g} M^{\mathrm{gr}}\left(\mathcal{G}_{1}\right) \cap \bigcap_{i=1}^{k} \operatorname{ker}\left(\ell_{i}-v_{i}\right), \\
\tau(\mathcal{G})=\tau\left(\mathcal{G}_{0}\right) \times_{g} \tau\left(\mathcal{G}_{1}\right) \cap \bigcap_{i=1}^{k} \operatorname{ker}\left(\ell_{i}-v_{i}\right) .
\end{gathered}
$$

We consider the lattice $M_{\mathrm{amb}}^{\mathrm{gr}}$ and the cone $\tau_{\mathrm{amb}}$, which are the combinatorial data of the ambient space $\mathbb{P}_{g\left(\mathcal{G}_{0}\right), n\left(\mathcal{G}_{0}\right)}$ described by Theorem 2.35, Example 2.36 and Ex- 
ample 2.37. The inclusion $X\left(\mathcal{G}_{0}\right) \subset \mathbb{P}_{g\left(\mathcal{G}_{0}\right), n\left(\mathcal{G}_{0}\right)}$ yields maps: $M_{\mathrm{amb}}^{\mathrm{gr}} \rightarrow M^{\mathrm{gr}}\left(\mathcal{G}_{0}\right)$ and $\tau_{\mathrm{amb}} \rightarrow \tau\left(\mathcal{G}_{0}\right)$. Forms $v_{i}, i=1, \ldots k$ pull-back to $M_{\mathrm{amb}}^{\mathrm{gr}}$ and we denote them by $\tilde{v}_{i}$, respectively. Now we define

$$
\begin{gathered}
M_{\mathcal{Y}}^{\mathrm{gr}}=M_{\mathrm{amb}}^{\mathrm{gr}}\left(\mathcal{G}_{0}\right) \times{ }_{g} M^{\mathrm{gr}}\left(\mathcal{G}_{1}\right) \cap \bigcap_{i=1}^{k} \operatorname{ker}\left(\ell_{i}-\tilde{v}_{i}\right), \\
\tau_{\mathcal{Y}}=\tau_{\mathrm{amb}}\left(\mathcal{G}_{0}\right) \times{ }_{g} \tau\left(\mathcal{G}_{1}\right) \cap \bigcap_{i=1}^{k} \operatorname{ker}\left(\ell_{i}-\tilde{v}_{i}\right) .
\end{gathered}
$$

We define a toric variety $\mathcal{Y}=\operatorname{Proj} \mathbb{C}[\tau \mathcal{Y}]$. Since the good quotient is a categorical quotient, by the construction we have the embedding

$$
X(\mathcal{G}) \hookrightarrow \mathcal{Y}
$$

Lemma 3.3 The inclusions

$$
M_{\mathcal{Y}}^{\mathrm{gr}} \hookrightarrow M_{\mathrm{amb}}^{\mathrm{gr}} \times M^{\mathrm{gr}}\left(\mathcal{G}_{1}\right) \quad \text { and } \quad \tau \mathcal{Y} \hookrightarrow \tau_{\mathrm{amb}} \times \tau\left(\mathcal{G}_{1}\right)
$$

induce a rational map

$$
\mathbb{P}_{g\left(\mathcal{G}_{0}\right), n\left(\mathcal{G}_{0}\right)} \times X\left(\mathcal{G}_{1}\right) \rightarrow \mathcal{Y}
$$

which is a good quotient map (of the set over which it is defined) with respect to the action of the $k$-dimensional torus $\mathbb{T}_{0}$ generated by one-parameter groups $\lambda_{v_{i}-\ell_{i}}$, where $i=1, \ldots k$. The subvariety

$$
\widehat{\mathcal{X}}=\mathcal{X}^{0} \times X\left(\mathcal{G}_{1}\right) \hookrightarrow \mathcal{B} \times \mathbb{P}^{7} \times X\left(\mathcal{G}_{1}\right)
$$

is $\mathbb{T}_{0} \times \mathbb{T}\left(X\left(\mathcal{G}_{1}\right)\right)$-invariant and its quotient $\mathcal{X}$ is locally complete intersection in $\mathcal{B} \times \mathcal{Y}$.

Proof The map given by inclusions of cones and lattices is a good quotient map by Theorem 1.14. Invariance of the resulting subvariety $\widehat{\mathcal{X}}$ follows by the invariance of $\mathcal{X}^{0} \hookrightarrow \mathcal{B} \times \mathbb{P}^{7}$ discussed in Example 3.1. The map is also clearly equivariant with respect to the big torus $\mathbb{T}\left(X\left(\mathcal{G}_{1}\right)\right)$ of $X\left(\mathcal{G}_{1}\right)$. Finally, since $\widehat{\mathcal{X}}$ is a complete intersection in $\mathcal{B} \times \mathbb{P}^{7} \times X\left(\mathcal{G}_{1}\right)$ its image $\mathcal{X}$ is a locally complete intersection in the quotient $\mathcal{B} \times \mathcal{Y}$. This follows from the definition of good quotient, which locally is an affine quotient [1, Chap. 5], hence functions defining $\widehat{\mathcal{X}}$ locally descend to functions defining $\mathcal{X}$.

Lemma 3.4 Over an open set $\mathcal{B}^{\prime} \subset \mathbb{P}^{2}$ containing points $[1,0,0],[0,1,0],[0,0,1]$ the projection morphism $\mathcal{X} \rightarrow \mathcal{B}^{\prime}$ is flat. The fibers over points $[1,0,0],[0,1,0]$, $[0,0,1]$ are reduced and isomorphic to, respectively, the geometric model of $\mathcal{G}$ and of its elementary mutations along the edge $e_{0}$.

Proof First we note that the fibers in question, $\mathcal{X}_{[*, *, *]}$, of $\mathcal{X} \rightarrow \mathcal{B}$ are geometric models as we claim. Indeed this follows from the universal properties of good quotients, 
cf. [1], as they are quotients of the respective products $\mathcal{X}_{[*, *, *]}^{0} \times X\left(\mathcal{G}_{1}\right)$, which are located, as three invariant subvarieties, in $\widehat{\mathcal{X}}=\mathcal{X}^{0} \times X\left(\mathcal{G}_{1}\right)$. This, in particular, implies that the respective fibers of $\mathcal{X} \rightarrow \mathcal{B}$ are of the expected dimension, hence they are contained in a set $\mathcal{B}^{\prime} \subset \mathbb{P}^{2}$ over which the map in question is equidimensional. Since $\mathcal{Y}$ is toric it is Cohen-Macaulay and because $\mathcal{X}$ is a locally complete intersection in $\mathcal{Y}$, it is Cohen-Macaulay too [4, Proposition 18.13]. Finally, the map $\mathcal{X} \rightarrow \mathcal{B}^{\prime}$ is equidimensional hence it is flat, because $\mathcal{B}^{\prime}$ is smooth, see [4, Theorem 18.16].

Theorem 3.5 Geometric models of connected trivalent graphs with the same number of leaves $n$ and cycles $g$ are deformation-equivalent in the projective toric variety $\mathbb{P}_{g, n}$, which is a quotient of $\mathbb{P}^{2^{n+2 g-1}-1}$ by a g-dimensional torus. Moreover, the deformations are invariant with respect to the action of the torus $\mathbb{T}(\mathcal{L})$ corresponding to the leaves of the graph.

Proof This is a combination of Proposition 2.10 and of Lemma 3.4. The $\mathbb{T}(\mathcal{L})$ equivariant property follows from Lemma 3.4 and Theorem 2.35; see also the discussion in the proof of Theorem 4.5.

Remark 3.6 Since the phylogenetic model of disjoint union of graphs is a product of the models of the pieces, see Lemma 2.21, we have proved that models of graphs with the same topological invariants are deformation-equivalent.

\section{Hilbert function of the model}

As we mentioned in Sect. 3, given a projective variety embedded in a projective space we have Hilbert function coming from the grading of the coordinates ring, or equivalently from the action of the one-dimensional torus $\mathbb{C}^{*}$. If our projective variety is equipped with an action of a bigger torus it is natural (see [8]) to consider a multigraded Hilbert function, whose domain consists of the characters of the torus.

We study multigraded Hilbert function of an embedded projective toric variety with the multigrading given by a subtorus of the big torus. For the graph model $X(\mathcal{G})$ the subtorus comes from a subset of leaves. We prove in Theorem 4.5 that the Hilbert function depends only on the topological invariants of the graph by showing that deformations constructed in the proof of Theorem 3.5 preserve the whole Hilbert series. As tools we use Lemma 4.3 to compute the Hilbert series of a torus invariant complete intersection. Lemma 4.4 gives the formula for the Hilbert series of a quotient of a toric variety by a subtorus of the big torus. We first state them in the algebraic setting.

\subsection{Ring with a torus action}

Let $R$ be a commutative $\mathbb{C}$-algebra with an action of a torus $\mathbb{T}$. Let $M_{\mathbb{T}}=\{\chi: \mathbb{T} \rightarrow$ $\left.\mathbb{C}^{*}\right\}$ denote the group of characters of the torus $\mathbb{T}$. Then we can write

$$
R=\bigoplus_{\chi \in M_{\mathbb{T}}} R_{\chi}
$$


as a sum of isotypical pieces indexed by the characters of the torus. We assume that each $R_{\chi}$ has finite dimension over $\mathbb{C}$. Then its Hilbert function $H_{R, \mathbb{T}}: M_{\mathbb{T}} \rightarrow \mathbb{N}$ is

$$
H_{R, \mathbb{T}}(\chi):=\operatorname{dim} R_{\chi}
$$

The Hilbert series is the generating series of $H_{R, \mathbb{T}}$

$$
h_{R, \mathbb{T}}(t):=\sum_{\chi \in M_{\mathbb{T}}} \operatorname{dim} R_{\chi} \cdot t^{\chi}
$$

Lemma 4.1 Let $R=\bigoplus_{\chi \in M_{\mathbb{T}}} R_{\chi}$ be a ring with a torus action. If $f_{1}, \ldots, f_{q}$ are homogeneous with $f_{i} \in R_{\chi_{i}}$ and form a regular sequence in $R$, then

$$
h_{R /\left\langle f_{1}, \ldots, f_{q}\right\rangle, \mathbb{T}}(t)=h_{R}(t) \cdot\left(1-t^{\chi_{1}}\right) \cdots\left(1-t^{\chi_{q}}\right) .
$$

Proof The statement for the single graded Hilbert series is explicitly given in [19, Corollary 3.2]. Its multigraded, more general, version with minor additional assumption can be found in [14, Claim 13.38]. The lemma follows by induction on the length of the regular sequence. For any homogeneous $f \in R_{\chi_{f}}$ which is not a zero divisor in $R$ and any $\chi \in M$ we have the exact sequence of $\mathbb{T}$-modules

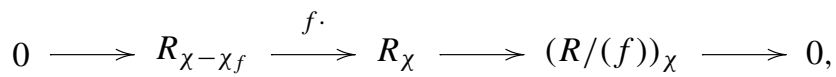

which implies that

$$
H_{R /(f)}(\chi)=\operatorname{dim}(R /(f))_{\chi}=\operatorname{dim} R_{\chi}-\operatorname{dim} R_{\chi-\chi_{f}}=H_{R}(\chi)-H_{R}\left(\chi-\chi_{f}\right) .
$$

This is equivalent to the required equality for Hilbert series.

Given a subtorus $\iota: \mathbb{S} \hookrightarrow \mathbb{T}$ we have the corresponding epimorphism of the character groups $\iota^{*}: M_{\mathbb{T}} \rightarrow M_{\mathbb{S}}$ and we can form the $\mathbb{S}$-invariant subring $R^{\mathbb{S}}$ of $R$ equipped with the residual action of the quotient torus $\mathbb{T} / \mathbb{S}$

$$
R^{\mathbb{S}}=\bigoplus_{\chi \in M_{\mathbb{T} / \mathbb{S}}} R_{\chi} .
$$

Then we have the following formula for the Hilbert series.

Lemma 4.2 Let $R$ be a ring equipped with an action of a torus $\mathbb{T}$ and let $\iota: \mathbb{S} \hookrightarrow \mathbb{T}$ be a subtorus. Then the Hilbert series of the invariant ring $R^{\mathbb{S}}$ is

$$
h_{R^{\mathbb{S}}, \mathbb{T} / \mathbb{S}}=\sum_{\chi \in \operatorname{ker} \iota^{*}} t^{\chi} \operatorname{dim} R_{\chi},
$$

where $\iota^{*}: M_{\mathbb{T}} \rightarrow M_{\mathbb{S}}$ is the dual map of the character groups. 


\subsection{Toric variety with a distinguished subtorus}

Let $X=\operatorname{Proj} R$ be a projective toric variety of dimension $d$ with an ample Weil divisor $\mathcal{L}$ where $R=\bigoplus_{m \in \mathbb{N}} R_{m}=\bigoplus_{m \in \mathbb{N}} H^{0}(X, m \mathcal{L})$ as in Sect. 1.3. Then $R$ has an action of a $d+1$ dimensional torus which is the product of the $d$-dimensional torus $\mathbb{T}$ of $X$ and the $\mathbb{C}^{*}$ from the grading. Any subtorus $\mathbb{S} \hookrightarrow \mathbb{T}$ of dimension $r$ induces a $\mathbb{Z}^{r+1}$-sub-grading. Then its multigraded Hilbert function $H_{X, \mathbb{S}}: M_{\mathbb{C}^{*} \times \mathbb{S}} \rightarrow \mathbb{N}$ with respect to $\mathbb{S}$ is

$$
H_{X, \mathbb{S}}(\chi):=H_{R, \mathbb{S}}(\chi)=\operatorname{dim} R_{\chi} .
$$

The generating series of $h_{R, \mathbb{S}}$ is the multigraded Hilbert series with respect to $\mathbb{S}$

$$
h_{X, \mathbb{S}}(t):=h_{R}(t)=\sum_{\chi \in M_{\mathbb{S}}} \operatorname{dim} R_{\chi} \cdot t^{\chi} .
$$

We have the following corollary of Lemma 4.1.

Corollary 4.3 Let $Y=\operatorname{Proj} R$ be a projective toric variety with an action of an $r$ dimensional subtorus $\mathbb{S} \subset \mathbb{T}$ of the big torus. Let us assume that $X \subset Y$ is an $\mathbb{S}$ invariant complete intersection in $Y$ given by the ideal $I(X)=\left\langle f_{1}, \ldots, f_{q}\right\rangle$, where $\operatorname{deg} f_{i}=\chi_{i}$. Then the $\mathbb{S}$-graded Hilbert series of $X$ is

$$
h_{X, \mathbb{S}}(t)=h_{Y}(t) \cdot\left(1-t^{\chi_{1}}\right) \cdots \cdot\left(1-t^{\chi_{q}}\right) \text {. }
$$

The next statement is a corollary of Lemma 4.2 by using the description of the quotient as the spectrum of invariants given in Theorem 1.6.

Lemma 4.4 Let $X=\operatorname{Proj} R$ be a projective toric variety with a subtorus $\iota: \mathbb{S} \hookrightarrow \mathbb{T}$ of the big torus as before. We assume that both actions are linearized with respect to the ample Weil divisor $\mathcal{L}$. Let $\iota^{*}: M_{\mathbb{T}} \rightarrow M_{\mathbb{S}}$ be the corresponding surjection of character lattices. Then

$$
h_{X / / \mathbb{S}}\left(t_{0}, t_{1}, \ldots, t_{r}\right)=\sum_{\chi \in \tau(X) \cap \mathrm{ker} \iota^{*}} t^{\chi} \cdot \operatorname{dim} R_{\chi} .
$$

We combine the above facts to get the equality of the Hilbert series of models of mutation-equivalent graphs.

Theorem 4.5 Let $\mathcal{G}_{1}$ and $\mathcal{G}_{2}$ be mutation-equivalent graphs and $S_{1}$ (respectively, $\mathcal{S}_{2}$ ) be a subset of leaves of $\mathcal{G}_{1}$ (respectively, $\mathcal{S}_{2}$ ). Assume that $\left|\mathcal{S}_{1}\right|=\left|\mathcal{S}_{2}\right|$. Then the multigraded series with respect to the tori associated to those sets of leaves are equal

$$
h_{X\left(\mathcal{G}_{1}\right), \mathbb{T}\left(\mathcal{S}_{1}\right)}=h_{X\left(\mathcal{G}_{2}\right), \mathbb{T}\left(\mathcal{S}_{2}\right)} .
$$

Proof Since they are mutation-equivalent by Lemma 2.10 we can assume the sequence of mutation takes the set $\mathcal{S}_{1}$ to the set $\mathcal{S}_{2}$. We can assume $\mathcal{G}_{1}$ and $\mathcal{G}_{2}$ differ by one mutation. In Sect. 3.2 we have constructed a flat family which is a complete 
intersection having the models $X\left(\mathcal{G}_{1}\right)$ and $X\left(\mathcal{G}_{2}\right)$ as fibers. Because both those models are complete intersections of the same type in the same ambient space by using Lemma 4.3 we conclude that the Hilbert series are equal.

We illustrate Theorem 4.5 on examples.

Example 4.6 We compute the Hilbert series for the models of graphs with two leaves and the first Betti number one $X(9)$ and $X(-\circ)$. As we saw in Example 2.30, using the notation of Example 2.36, the cone $\tau$ ( $(\mathcal{X})$ has generators of the following multidegrees:

\begin{tabular}{llllllll}
\hline Coordinate & $x_{0000}$ & $x_{1100}$ & $x_{0011}$ & $x_{1111}$ & $y_{1}$ & $z_{1}$ & $z_{2}$ \\
\hline Degree & $(1,0,0)$ & $(1,0,0)$ & $(1,1,1)$ & $(1,1,1)$ & $(2,1,1)$ & $(2,2,0)$ & $(2,0,2)$ \\
\hline
\end{tabular}

As a consequence $X(\mathcal{X})$ is a complete intersection in $\mathbb{P}\left(1^{4}, 2^{3}\right)$ of a quadric $x_{1100} x_{0011}-x_{0000} x_{1111}$ of multidegree $(2,1,1)$ and a quartic $y_{1}^{2}-z_{1} z_{2}$ of multidegree $(4,2,2)$. Thus

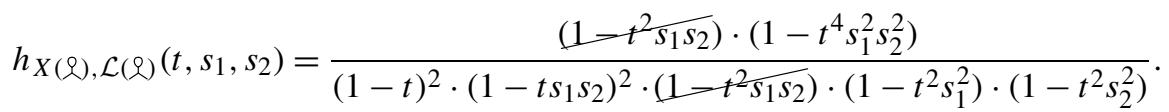

Ignoring the multigrading by the two-dimensional torus spanned by leaves (by setting $s_{1}=s_{2}=1$ ) we get

$$
h_{X(\rho)}(t)=\frac{\left(1-t^{2}\right) \cdot\left(1-t^{4}\right)}{(1-t)^{4} \cdot\left(1-t^{2}\right)^{3}}=\frac{1+t^{2}}{(1-t)^{4} \cdot\left(1-t^{2}\right)} \text {. }
$$

We have seen in Example 2.37 that $\tau(-\circ)$ has six generators: those are the elements in the above table for 9 apart from $y_{1}$. The model $X(-\odot)$ is a hypersurface of degree $(4,2,2)$ in $\mathbb{P}\left(1^{4}, 2^{2}\right)$ so

$$
\begin{aligned}
h_{X(-\odot), \mathcal{L}(-\odot)}\left(t, s_{1}, s_{2}\right) & =\frac{\left(1-t^{4} s_{1}^{2} s_{2}^{2}\right)}{(1-t)^{2} \cdot\left(1-t s_{1} s_{2}\right)^{2} \cdot\left(1-t^{2} s_{1}^{2}\right) \cdot\left(1-t^{2} s_{2}^{2}\right)} \\
& =h_{X(\text { (), } \mathcal{L} \text { (९) }}\left(t, s_{1}, s_{2}\right) .
\end{aligned}
$$

Again we can ignore the multigrading and get

$$
h_{X(-))}(t)=\frac{\left(1-t^{4}\right)}{(1-t)^{4} \cdot\left(1-t^{2}\right)^{2}}=\frac{1+t^{2}}{(1-t)^{4} \cdot\left(1-t^{2}\right)} .
$$

We expand to see the first few terms

$$
h_{X(\text { ( ) }}(t)=1+4 t+12 t^{2}+28 t^{3}+57 t^{4}+104 t^{5}+176 t^{6}+280 t^{7}+O\left(t^{8}\right) \text {. }
$$


Fig. 24 Caterpillar graph

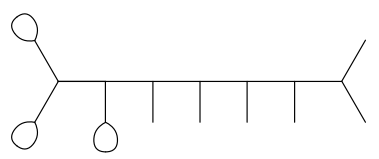

Example 4.7 The Hilbert series of models of both graphs $\Theta$ and $\$$ with no leaves (thus no additional grading) and two cycles is

$$
\begin{aligned}
h_{X(8)}(t)=h_{X(\ominus)}(t)= & \frac{1}{\left(t^{4}-4 t^{3}+6 t^{2}-4 t+1\right)} \\
= & 1+4 t+10 t^{2}+20 t^{3}+35 t^{4}+56 t^{5} \\
& +84 t^{6}+120 t^{7}+O\left(t^{8}\right) .
\end{aligned}
$$

This is because $X(\Theta)$ is $\mathbb{P}^{3}=\left(\mathbb{P}^{3} \times \mathbb{P}^{3}\right) / /\left(\mathbb{C}^{*} \times \mathbb{C}^{*} \times \mathbb{C}^{*}\right)$.

\subsection{Computing the Hilbert function}

Given a trivalent tree $\mathcal{T}$ with $n$ leaves we computed the Hilbert function $H_{X(\mathcal{T})}$ of its model in [3] as

$$
H_{X(\mathcal{T}), \mathbb{S}(\{l\})}(m, k)=1_{m}^{\star n}(k)
$$

where the additional grading corresponds to a distinguished leaf $l$, $\star$ is an appropriate summing formula and $1_{m}$ is the constant function. This inductive formula for $H_{X(\mathcal{T})}$ uses the decomposition of the tree $\mathcal{T}$ as a sum of tripods, which leads to the presentation of the polytope $\Delta(\mathcal{T})$ as a fiber product of tetrahedrons $\Delta($ 人).

The same method works for any trivalent graph. We proved in Theorem 4.5 that the Hilbert functions of mutation-equivalent graphs are equal. By Lemma 2.10 we know that any graph is mutation-equivalent to a graph of the shape depicted in Fig. 24.

This means we have reduced the calculation to the case of caterpillar graphs. As we have described in Sect. 2.1, any graph is presented as union of tripods $入$ with identifications. More precisely, any trivalent graph is built from $ᄉ$ by the operations of grafting two graphs and gluing two leaves.

Remark 4.8 To produce a caterpillar graph $\mathcal{G}$ from |'s (leaves) and Y's (leaves with loop) using $\star$ and $\supset$, we need the second operation only once per graph and only in the case when $\mathcal{G}$ has no leaves.

Remark 4.9 On the level of graph models we have

$$
X\left(\mathcal{G}_{1} \star \mathcal{G}_{2}\right)=\left(X\left(\mathcal{G}_{1}\right) \times X(\lambda) \times X\left(\mathcal{G}_{2}\right)\right) / /\left(\mathbb{C}^{*}\right)^{2}
$$


and

$$
X\left(\mathcal{G}_{l_{2}}^{l_{1}} \supset\right)=X(\mathcal{G}) / / \mathbb{C}^{*}
$$

where the actions of the tori were described in Sect. 2.4.

We give a formula for $h_{X\left(\mathcal{G}_{1} \star \mathcal{G}_{2}\right), \mathbb{S}\left(\mathcal{L}\left(\mathcal{G}_{1} \star \mathcal{G}_{2}\right)\right)}$ and for $h_{X\left(\mathcal{G}_{l_{2}}^{l_{1}} \supset\right), \mathbb{S}\left(\mathcal{L}\left(\mathcal{G}_{l_{2}}^{l_{1}} \supset\right)\right.}$, using the above fact about how the model of $\mathcal{G}_{1} \star \mathcal{G}_{2}$ is constructed from smaller pieces.

$$
\begin{aligned}
h_{X}\left(\mathcal{G}_{1} \star \mathcal{G}_{2}\right), \mathbb{S}\left(\mathcal{L}\left(\mathcal{G}_{1} \star \mathcal{G}_{2}\right)\right) & =h_{X}\left(\mathcal{G}_{1}\right), \mathbb{S}\left(\mathcal{L}\left(\mathcal{G}_{1}\right)\right) \star h_{X}\left(\mathcal{G}_{2}\right), \mathbb{S}\left(\mathcal{L}\left(\mathcal{G}_{2}\right)\right) \\
:= & \text { the part containing monomials of the form }\left(t_{1} t_{2} t_{3}\right)^{i}\left(s^{\prime}\right)^{0}\left(s^{\prime \prime}\right)^{0}\left(s^{\prime \prime \prime}\right)^{j} s^{I} \text { of } \\
& h_{X\left(\mathcal{G}_{1}\right)}\left(t_{1}, s_{1}, \ldots, s_{n_{1}}, \frac{1}{s^{\prime}}\right) \cdot h_{X(\curlywedge)}\left(t_{3}, s^{\prime}, s^{\prime \prime}, s^{\prime \prime \prime}\right) \cdot h_{X\left(\mathcal{G}_{2}\right)}\left(t_{2}, s_{n_{1}+1}, \ldots, s_{n_{1}+n_{2}}, \frac{1}{s^{\prime \prime}}\right)
\end{aligned}
$$

where $s=\left(s_{1}, \ldots, s_{n_{2}}\right)$ and $I$ is the exponent vector.

Let us compute the input functions: apart from the constant one which corresponds to leaves of $\mathcal{G}$ we have $H_{X(9), S(\{l\})}$ the Hilbert function of the model of graph with two edges. Recall that the model $X(\mathcal{Y})$ is $\mathbb{P}^{3} / / \mathbb{C}^{*}=\mathbb{P}(1,1,2)$, where the $\mathbb{C}^{*}$ action has weights $\left[\begin{array}{llll}0 & 1 & 0 & -1\end{array}\right]$ on $\mathbb{P}^{3}$. Here is the list of generators with weights and the resulting graded Hilbert function

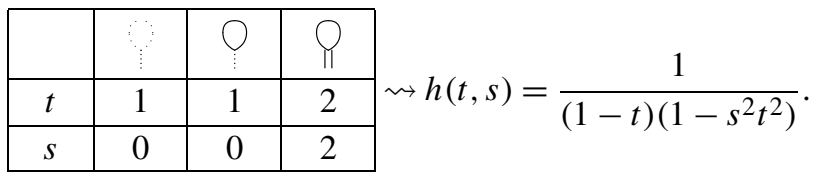

We can expand Formula (4.1), setting $f$ to be a Hilbert function of some graph, to get for $k \leq \frac{m}{2}$

$$
\begin{aligned}
h_{X(९)} \star f(k)= & (m-k+1) \sum_{a_{0}=0}^{m-k-1} f(a)[2 \mid k+a](a+1) \\
& +(k+1) \sum_{a_{0}=m-k}^{k} f(a)[2 \mid k+a](m+2-a) \\
& +k \sum_{a_{0}=k+1}^{m} f(a)[2 \mid k+a](m+1-a)
\end{aligned}
$$

and for $k \geq \frac{m}{2}$

$$
h_{X(\vartheta)} \star f(k)=(m-k+1) \sum_{a_{0}=0}^{m-k-1} f(a)[2 \mid k+a](a+1)
$$




$$
\begin{aligned}
& +(m-k+1) \sum_{a_{0}=m-k}^{k} f(a)[2 \mid k+a](2 m-2 k-a+1) \\
& +(m-k) \sum_{a_{0}=k+1}^{m} f(a)[2 \mid k+a](2 m-2 k-a)
\end{aligned}
$$

where $a=\left(a_{0}, a_{1}, \ldots, a_{n}\right)$.

In the same way we can write

$$
\begin{aligned}
& h_{X\left(\mathcal{G}_{l_{2}}^{\left.l_{1} \supset\right)}\right.}\left(t, s_{1}, \ldots, s_{n}\right) \\
& \quad=\text { the part that contains monomials } t^{i}\left(s^{\prime}\right)^{0} s^{I} \text { of } h_{X(\mathcal{G})}\left(t, s_{1}, \ldots, s_{n}, s^{\prime}, \frac{1}{s^{\prime}}\right)
\end{aligned}
$$

where $s=\left(s_{1}, \ldots, s_{n}\right)$ and $I$ is the exponent vector.

Acknowledgements Author was partially supported by Texas Advanced Research Grant No. 0103660054-2007.

Open Access This article is distributed under the terms of the Creative Commons Attribution Noncommercial License which permits any noncommercial use, distribution, and reproduction in any medium, provided the original author(s) and source are credited.

\section{References}

1. Białynicki-Birula, A.: Quotients by actions of groups. In: Algebraic Quotients. Torus Actions and Cohomology. The Adjoint Representation and the Adjoint Action. Encyclopedia Math. Sci., vol. 131, pp. 1-82. Springer, Berlin (2002)

2. Bosma, W., Cannon, J., Playoust, C.: The Magma algebra system. I. The user language. J. Symb. Comput. 24(3-4), 235-265 (1997). Computational algebra and number theory (London, 1993)

3. Buczyńska, W., Wiśniewski, J.A.: On geometry of binary symmetric models of phylogenetic trees. J. Eur. Math. Soc. 9(3), 609-635 (2007)

4. Eisenbud, D.: Commutative Algebra with a View Toward Algebraic Geometry. Graduate Texts in Mathematics, vol. 150. Springer, New York (1995)

5. Felsenstein, J.: Inferring Phylogenies. Sinauer Press, Sunderland (2004)

6. Fulton, W.: Introduction to Toric Varieties. Annals of Mathematics Studies, vol. 131. Princeton University Press, Princeton, NJ (1993). The William H. Roever Lectures in Geometry

7. Grothendieck, A.: Éléments de géometrie algébrique (rédigés avec la collaboration de Jean Dieudonné): II. Étude globale élémentaire de quelques classes de mophismes. Graduate Texts in Mathematics, vol. 52. Publications mathématique de l'IHÉS, Bures-sur-Yvette (1961)

8. Haiman, M., Sturmfels, B.: Multigraded Hilbert schemes. J. Algebr. Geom. 13(4), 725-769 (2004)

9. Hartshorne, R.: Algebraic Geometry. Graduate Texts in Mathematics, vol. 52. Springer, New York (1977).

10. Jeffrey, L.C., Weitsman, J.: Bohr-Sommerfeld orbits in the moduli space of flat connections and the Verlinde dimension formula. Commun. Math. Phys. 150(3), 593-630 (1992)

11. Manon, C.A.: The algebra of conformal blocks. arXiv:0910.0577v3 [math.AG] (2009)

12. Manon, C.A.: Coordinate rings for the moduli of $S L_{2}(\mathbb{C})$ quasi-parabolic principal bundles on a curve and toric fiber products. arXiv:1105.2045v1 [math.AC]

13. Mumford, D., Fogarty, J., Kirwan, F.: Geometric Invariant Theory, 3rd edn. Ergebnisse der Mathematik und ihrer Grenzgebiete (2) [Results in Mathematics and Related Areas (2)], vol. 34. Springer, Berlin (1994) 
14. Miller, E., Sturmfels, B.: Combinatorial Commutative Algebra. Graduate Texts in Mathematics, vol. 227. Springer, New York (2005)

15. Oda, T.: Convex Bodies and Algebraic Geometry. Ergebnisse der Mathematik und ihrer Grenzgebiete (3) [Results in Mathematics and Related Areas (3)], vol. 15. Springer, Berlin, (1988). An introduction to the theory of toric varieties, Translated from the Japanese

16. Pachter, L., Sturmfels, B.: Statistics. In: Algebraic Statistics for Computational Biology, pp. 3-42. Cambridge Univ. Press, New York (2005)

17. Reid, M.: Graded rings and birational geometry. In: Ohno, K. (ed.) Proceedings of Algebraic Geometry Symposium, Kinosaki, Oct. 2000, pp. 1-72 (2000)

18. Semple, C., Steel, M.: Phylogenetics. Oxford Lecture Series in Mathematics and Its Applications, vol. 24. Oxford University Press, Oxford (2003)

19. Stanley, R.P.: Hilbert functions of graded algebras. Adv. Math. 28(1), 57-83 (1978)

20. Sturmfels, B., Xu, Z.: Sagbi basis and Cox-Nagata rings. J. Eur. Math. Soc. 12(2), 429-459 (2010) 